\title{
Review \\ Jacobi Multipliers in Integrability and the Inverse Problem of Mechanics
}

\author{
José F. Cariñena ${ }^{1, *}$ and José Fernández-Núñez ${ }^{2}$ \\ 1 Departamento de Física Teórica and IUMA, Universidad de Zaragoza, 50009 Zaragoza, Spain \\ 2 Departamento de Física, Universidad de Oviedo, 33007 Oviedo, Spain; nonius@uniovi.es \\ * Correspondence: jfc@unizar.es
}

check for updates

Citation: Cariñena, J.F.;

Fernández-Núñez, J. Jacobi

Multipliers in Integrability and the Inverse Problem of Mechanics. Symmetry 2021, 13, 1413. https:// doi.org/10.3390/sym13081413

Academic Editors: Trong Tuong Truong and Youssef N. Raffoul

Received: 22 June 2021

Accepted: 30 July 2021

Published: 2 August 2021

Publisher's Note: MDPI stays neutral with regard to jurisdictional claims in published maps and institutional affiliations.

Copyright: (c) 2021 by the authors. Licensee MDPI, Basel, Switzerland. This article is an open access article distributed under the terms and conditions of the Creative Commons Attribution (CC BY) license (https:/ / creativecommons.org/licenses/by/ $4.0 /)$.

\begin{abstract}
We review the general theory of the Jacobi last multipliers in geometric terms and then apply the theory to different problems in integrability and the inverse problem for one-dimensional mechanical systems. Within this unified framework, we derive the explicit form of a Lagrangian obtained by several authors for a given dynamical system in terms of known constants of the motion via a Jacobi multiplier for both autonomous and nonautonomous systems, and some examples are used to illustrate the general theory. Finally, some geometric results on Jacobi multipliers and their use in the study of Hojman symmetry are given.
\end{abstract}

Keywords: Jacobi multipliers; inverse problem; nonstandard Lagrangians; integrability

PACS: 02.30.Hq; 02.30.Zz; 02.40.-k; 45.20.Jj

MSC: 70F17; 70G45; 70H03

\section{Introduction}

The Lagrangian approach to conservative systems originally tried to incorporate the existence of holonomic constraints by invariant under changes of coordinates and establishes, as a fundamental principle, the extremal property of the action for the motions. One usually considers Lagrangians of the so-called mechanical type where the Lagrangian function $L$ is the difference between a kinetic term and a potential term; however, a theory for a general form of the Lagrangian function has also been developed. The set of its corresponding Euler-Lagrange equations is a system of second-order differential equations (see any classical book on Classical Mechanics [1]):

$$
\frac{\partial L}{\partial q^{i}}=\frac{d}{d t}\left(\frac{\partial L}{\partial \dot{q}^{i}}\right), \quad i=1, \ldots n .
$$

In order to integrate such a system, knowledge of some of its first-integrals is very useful. For this aim, the analysis of the symmetries of the Lagrangian can be used via the well-known Noether theorem. On the other hand, by means of the Lagrangian function, we can achieve a Hamiltonian formulation and proceed to the quantization of the mechanical system. There are systems not only in Physics but also in other areas of Science, such as Biology, Chemistry, or Economy, whose evolution is described by second-order differential equations, and it would be of a great interest to know whether or not they are of the variational type, i.e., of the Lagrangian type. The fundamental point is the so-called inverse problem of mechanics, i.e., the determination, for a given system of second-order differential equations, of conditions for the existence of a Lagrangian function whose system of Euler-Lagrange equations is (equivalent to) the given system. The main result being given by Helmholtz [2,3]: More explicitly, given a system of second-order differential Equation (1),

$$
\ddot{q}^{i}=f^{i}(q, \dot{q}), \quad i=1, \ldots n,
$$


Helmholtz established in [2] the conditions for the existence of functions $g_{i j}(q, \dot{q})$, with $\operatorname{det}\left[g_{i j}\right] \neq 0$, and a regular Lagrangian $L$ such that $g_{i j}(q, \dot{q})\left(\ddot{q}^{j}-f^{j}(q, \dot{q})\right)=0$ (summation on repeated indices is understood) are the Euler-Lagrange equation system of the function $L$. In terms of the functions $f^{i}$, these Helmholtz conditions for the functions $g_{i j}$ are given (see $[4,5])$ as follows:

(i) $\quad g_{i j}=g_{j i}$

(ii) $\quad \operatorname{det}\left[g_{i j}\right] \neq 0$

(iii) $\frac{\partial g_{i j}}{\partial v^{k}}=\frac{\partial g_{i k}}{\partial v^{j}}$

(iv) $\quad \Gamma\left(g_{i j}\right)=\frac{1}{2} g_{k j} \frac{\partial f^{k}}{\partial v^{i}}+\frac{1}{2} g_{i k} \frac{\partial f^{k}}{\partial v^{j}}$

(v) $\quad g_{i k}\left[\frac{\partial f^{k}}{\partial q^{j}}+\frac{1}{4} \frac{\partial f^{k}}{\partial v^{l}} \frac{\partial f^{l}}{\partial v^{j}}-\frac{1}{2} \Gamma\left(\frac{\partial f^{k}}{\partial v^{j}}\right)\right]=g_{j k}\left[\frac{\partial f^{k}}{\partial q^{i}}+\frac{1}{4} \frac{\partial f^{k}}{\partial v^{l}} \frac{\partial f^{l}}{\partial v^{i}}-\frac{1}{2} \Gamma\left(\frac{\partial f^{k}}{\partial v^{i}}\right)\right]$,

where $\Gamma$ is the vector field

$$
\Gamma(q, v)=v^{i} \frac{\partial}{\partial q^{i}}+f^{i}(q, v) \frac{\partial}{\partial v^{i}} .
$$

In the affirmative case, these five properties lead to the existence of a function $L$ such that the $g_{i j}$ takes the form

$$
g_{i j}=\frac{\partial^{2} L}{\partial v^{i} \partial v^{j}}
$$

The non-uniqueness of such Lagrangian functions and the implications of the existence of alternative Lagrangians is also a relevant point: in some particular cases, there exist several different (and non gauge equivalent) solutions. In these cases, two alternative Lagrangians can be used to construct constants of the motion, as proven in [6] for the one-dimensional case and generalised in [7] for the multidimensional case (see also [8] and references therein for a geometric approach). The geometric approach to Lagrangian mechanics developed during the last forty years [4,9-12], which allows treating the preceding question, suggests the use of more general Lagrangian functions, sometimes called nonstandard Lagrangians, for instance, for second-order Riccati and Abel equations [13,14]. This subject has received a lot of attention during the last few years, and many applications have been developed [15-20]. The simplest case is that of a one-dimensional configuration space, i.e., one second-order differential equation, and then the Helmholtz criterion reduces to the existence of a function satisfying a differential equation, which turns out to be that of a Jacobi last multiplier [21]. In fact, in the simpler one-dimensional case, the inverse problem amounts to determine for a given second-order differential equation, $\ddot{x}=F(x, \dot{x}, t)$, a pair of functions, $g$ and $L$, such that

$$
g(x, \dot{x}, t)(\ddot{x}-F(t, x, \dot{x}))=\frac{d}{d t}\left(\frac{\partial L}{\partial \dot{x}}\right)-\frac{\partial L}{\partial x}=\frac{\partial^{2} L}{\partial \dot{x}^{2}} \ddot{x}+\frac{\partial^{2} L}{\partial x \partial \dot{x}} \dot{x}+\frac{\partial^{2} L}{\partial \dot{x} \partial t}-\frac{\partial L}{\partial x} .
$$

Therefore, the function $g$ must be $g=\partial^{2} L / \partial \dot{x}^{2}$, and furthermore,

$$
g F=\frac{\partial L}{\partial x}-\frac{\partial^{2} L}{\partial x \partial \dot{x}} \dot{x}-\frac{\partial^{2} L}{\partial \dot{x} \partial t} .
$$

Taking derivatives with respect to $\dot{x}$ in both members, we find as a necessary condition

$$
\frac{\partial}{\partial \dot{x}}(g F)=-\frac{\partial g}{\partial x} \dot{x}-\frac{\partial g}{\partial t}
$$

which can be rewritten as

$$
\frac{d g}{d t}+g \frac{\partial F}{\partial \dot{x}}=0
$$


This equation explicitly shows that the function $g$ must be a Jacobi multiplier, a concept introduced long time ago by Jacobi $[22,23]$ but forgotten for a long time in the physical literature, and which is receiving attention in the last few years, and actually, its use has clarified many interesting points in classical mechanics thanks to the works by Leach, Nucci and coworkers [24-26].

A method for the search of a Hamiltonian formulation of a given second-order differential equation has recently been developed in [27]. Of course, such a Hamiltonian formulation is not uniquely determined. Actually, the Hamiltonian and the corresponding formulation is an auxiliary tool in the search for the solution of the given equation, and the same can be said of a Lagrangian formulation. Even the stronger concept of gauge equivalence of two Lagrangian does not lead to the same Hamiltonian, and it is only the motions of configuration space coordinates in both descriptions that coincide, but it is not true for the new auxiliary variables, that is, the momenta. In the Hamiltonian approach, given a solution $H$, we can obtain a new Hamiltonian formulation by making use of a fouling transformation $[6,28]$. Moreover, the corresponding quantum model depends critically on the choice of the Hamiltonian description.

The method proposed in [27] is an ad hoc method, and it is not fully justified because it is not clear how to use the implicit function theorem in the formula preceding (2.4) in [27] to find the differentiable function $I\left(x, x^{\prime}\right)$. However, the theory of Jacobi multipliers [22,23,29,30], which was developed to integrate a system of first-order differential equations by quadratures when some first-integrals and a Jacobi multiplier are known, can be extended to systems of second-order differential equations from a geometric perspective because if a system of first-order differential equation is replaced by a vector field in a manifold, whose integral curves are the solution of the system, in a similar way, a given system of second-order differential equations corresponds to a vector field on its tangent bundle of a special class of vector fields. In the particular case of a second-order differential equation, the theory provides a solution for the so-called inverse problem of mechanics because it asserts the existence of a Lagrangian description for the given equation. The rôle of the function $I\left(x, x^{\prime}\right)$ appearing in [27] is that of a first-integral (see (2.2) in [27]), which may be used to determine a Jacobi multiplier and then, as a consequence, a Lagrangian formulation. This is a well-established procedure, which should replace the incomplete derivation of Section 2 in [27].

As the theory of Jacobi multipliers is not well known in physics, in spite of its many applications, the aim of this paper is to give a short updated review of this concept from a geometric perspective (see also [31-33]) and show by means of illustrative examples some of its physical applications. The concept of Jacobi multipliers is introduced in Section 2, where the particular case of a two-dimensional autonomous system of first-order ordinary differential equations illustrates the theory. Following with these lower dimensional instances, Jacobi multipliers for the simple case of an autonomous second-order differential equation and the relation with the inverse problem is analysed in Section 3, and, as an important application in physics, the theory will be used to re-derive, in Section 4 , the explicit form of a Lagrangian for a dynamics with a given constant of the motion proposed in [27,34-38]. In order for the paper be self-contained, we have added, in Section 5, a short summary with the fundamental results of the theory of symplectic manifolds and its applications in the geometric approach to Hamiltonian and Lagrangian mechanics. The results of Section 4 are generalised and extended in Section 6 to the non-autonomous cases, with illustrative examples given in Section 7. As divergence-free vector fields enjoy interesting integrability properties, a remarkable relationship between an infinitesimal symmetry and a Jacobi multiplier with applications in integrability is analysed in Section 8. Finally, Section 9 is devoted to point out the usefulness of Jacobi multipliers in a method for finding constants of motion that generalises the so-called Hojman symmetry. 


\section{Jacobi Last Multipliers}

Modern tools of differential geometry have been shown to be very efficient for a better understanding of differential equations and dynamical systems. Instead of using affine spaces, one needs to consider the more general concept of differentiable manifold, and systems of first-order differential equations are replaced by vector fields. Then, the associated system of differential equations is a way of expressing the set of integral curves of such a vector field that appear as solutions of such a system in local coordinates. Additional geometric structures on the manifold, satisfying some compatibility conditions, particularly invariant structures, are very useful in the integrability of the system. Recall that if $(M, \Omega)$ is a $n$-dimensional-oriented manifold, and $X \in \mathfrak{X}(M)$ is a vector field, then the Lie derivative $\mathcal{L}_{X} \Omega$ is proportional to $\Omega$, and we call the divergence of $X$ the function div $X$ defined by

$$
\mathcal{L}_{X} \Omega=\operatorname{div} X \Omega,
$$

or, equivalently, by $d(i(X) \Omega)=\operatorname{div} X \Omega$ because the $n$-form $\Omega$ is closed. Hence, as for each function $f \in C^{\infty}(M), \mathcal{L}_{X}(f \Omega)=d(i(X)(f \Omega))=d(i(f X) \Omega)=\mathcal{L}_{f X} \Omega$, we see, using the properties of Lie derivatives, that

$$
\operatorname{div}(f X)=f \operatorname{div} X+X(f) .
$$

In local coordinates $\left(x^{1}, \ldots, x^{n}\right)$ such that $\Omega=d x^{1} \wedge \cdots \wedge d x^{n}$, if $X$ is the vector field with local expression,

$$
X(x)=X^{i}(x) \frac{\partial}{\partial x^{i}}
$$

we recover the usual expression for the divergence of $X$ (recall that summation on repeated indices is understood):

$$
\operatorname{div} X=\frac{\partial X^{i}}{\partial x^{i}}
$$

Vector fields $X \in \mathfrak{X}(M)$ such that $\operatorname{div} X=0$ are said to be divergence-free vector fields. In the Euclidean three-dimensional case, they are called solenoidal vector fields. The concept of Jacobi multiplier for a vector field $X$ in an oriented manifold $(M, \Omega)$ introduced by Jacobi $[22,23]$ is also very helpful to understand some aspects of the inverse problem of Lagrangian mechanics:

Definition 1. A nonvanishing function $R \in C^{\infty}(M)$ in an oriented manifold $(M, \Omega)$ is said to be a Jacobi multiplier for $X \in \mathfrak{X}(M)$ if the $(n-1)$-form $R(i(X) \Omega)$ is closed. In other words, there exists a (locally defined) $(n-2)$-form $\sigma$ such that

$$
R(i(X) \Omega)=d \sigma .
$$

Note that this condition is equivalent to saying the vector field $R X$ is divergencefree, i.e.,

$$
\operatorname{div}(R X)=0,
$$

because

$$
\mathcal{L}_{R X} \Omega=d(i(R X) \Omega)=d(R i(X) \Omega),
$$

and therefore, Equation (6) implies that $\mathcal{L}_{R X} \Omega=0$. Thus, a vector field $X$ such that $\operatorname{div} X \neq 0$ can be transformed into a divergence-free one spanning the same one-dimensional distribution by multiplication by a Jacobi multiplier for $X$. This divergence-free nature allows us to reduce the number of symmetries needed to the integration process by quadratures, see, e.g., $[22,23,39]$. However, in this article, we are particularly interested in the relevance of the concept in the search of a Lagrangian formulation for the simpler case of a given secondorder differential equation. This may be very important in the search of a possible analogous quantum system because the traditional quantisation process uses a Hamiltonian quantisation, or even the Feynman path-integral quantisation is based on a Lagrangian formulation. 
Alternatively, from the above-mentioned property $\mathcal{L}_{X}(R \Omega)=\mathcal{L}_{R X} \Omega$, we see that $R$ is a Jacobi multiplier for $X$ if and only if the vector field $X$ is divergence-free with respect to $R \Omega$, i.e., we see the equivalence of searching for Jacobi multipliers on one side and the more geometric concept of invariant volume-forms on the other: a volume form $\Omega^{\prime}=R \Omega$ is $X$-invariant, i.e., $\mathcal{L}_{X} \Omega^{\prime}=0$ if and only if $R$ is a Jacobi multiplier for the vector field $X$ in the oriented manifold $(M, \Omega)$. Moreover, this property also shows that $R$ is a Jacobi multiplier for $X$ in the oriented manifold $(M, \Omega)$ if and only if $f R$ is a Jacobi multiplier for $X$ in the oriented manifold $\left(M, f^{-1} \Omega\right)$ for each positive function $f$.

As another consequence of the property, the set of vector fields admitting $R$ as a Jacobi multiplier is a real Lie subalgebra because from $\mathcal{L}_{X}(R \Omega)=\mathcal{L}_{Y}(R \Omega)=0$, as $\mathcal{L}_{X+\lambda Y}(R \Omega)=\mathcal{L}_{X}(R \Omega)+\lambda \mathcal{L}_{Y}(R \Omega)$, for $\lambda \in \mathbb{R}$, we see that $\mathcal{L}_{X+\lambda Y}(R \Omega)=0$, and then, it is a $\mathbb{R}$-linear space, and, moreover, from the relation for Lie derivatives of forms $\mathcal{L}_{X} \circ \mathcal{L}_{Y}-\mathcal{L}_{Y} \circ \mathcal{L}_{X}=\mathcal{L}_{[X, Y]}$, it also follows that

$$
\mathcal{L}_{[X, Y]}(R \Omega)=\mathcal{L}_{X} \mathcal{L}_{Y}(R \Omega)-\mathcal{L}_{Y} \mathcal{L}_{X}(R \Omega)=0 .
$$

Another relevant geometric relation is that for any arbitrary pair $X, Y$ of vector fields in an oriented manifold $(M, \Omega)$, we have that

$$
\mathcal{L}_{X}(\operatorname{div}(Y))-\mathcal{L}_{Y}(\operatorname{div}(X))=\operatorname{div}([X, Y]),
$$

which follows from the above-mentioned relation by taking the difference of $\mathcal{L}_{X}\left(\mathcal{L}_{Y} \Omega\right)=$ $\mathcal{L}_{X}(\operatorname{div}(Y) \Omega)=\mathcal{L}_{X}(\operatorname{div}(Y)) \Omega+\operatorname{div}(Y) \operatorname{div}(X) \Omega$ and the corresponding relation $\mathcal{L}_{Y}\left(\mathcal{L}_{X} \Omega\right)=\mathcal{L}_{Y}(\operatorname{div}(X)) \Omega+\operatorname{div}(X) \operatorname{div}(Y) \Omega$.

Of course, in local coordinates such that $\Omega=d x^{1} \wedge \cdots \wedge d x^{n}$, the condition for $R$ to be a Jacobi multiplier for the vector field $X$ given by Equation (5) is

$$
\frac{\partial\left(R X^{i}\right)}{\partial x^{i}}=0,
$$

which is but the local coordinate expression of condition given in Equation (7) for the multiplier $R$, i.e.,

$$
X(R)+R \operatorname{div} X=0 .
$$

Note that this implies that if $X$ is divergence-free, then $R$ is a Jacobi multiplier if and only if $R$ is a first-integral. More generally, if $R$ is a Jacobi multiplier for the vector field $X$, then $f R$ is another multiplier if and only if $f$ is a nonvanishing first-integral of the vector field $X$ because if Equation (10) holds, then replacing $R$ by $f R$ on the left hand side of Equation (10), we obtain

$$
(f R) \operatorname{div} X+X(f R)=f(R \operatorname{div} X+X(R))+R X(f)=R X(f),
$$

and, consequently, $f R$ is a Jacobi multiplier if and only if $X(f)=0$. Therefore, a vector field may admit an infinite number of Jacobi multipliers.

In the lower dimensional case of an autonomous system of first-order ordinary differential equations

$$
\left\{\begin{array}{l}
\dot{x}=P(x, y) \\
\dot{y}=Q(x, y)
\end{array}\right.
$$

whose solutions are the integral curves of the associated vector field on $\mathbb{R}^{2}$

$$
X=P(x, y) \frac{\partial}{\partial x}+Q(x, y) \frac{\partial}{\partial y},
$$

the corresponding phase-flow is given by the solutions of the differential equation

$$
\frac{d y}{d x}=\frac{Q(x, y)}{P(x, y)},
$$


and then any other vector field proportional to $X$ gives rise to the same flow, i.e., to the previous differential Equation (13), which is described by the one-dimensional distribution generated by $X$. Such a distribution can also be defined by the 1 -form

$$
\alpha=P(x, y) d y-Q(x, y) d x
$$

because $\alpha(X)=0$ or by any other proportional one. The integral curves, $\gamma: I \rightarrow \mathbb{R}^{2}$, $\gamma(t)=(x(t), y(t))$, of the distribution defined by $\alpha$ are those such that $\gamma^{*} \alpha=0$, and as

$$
\gamma^{*} \alpha=(P(x, y) \dot{y}-Q(x, y) \dot{x}) d t=0,
$$

the one-dimensional distribution determined by $\operatorname{Ker} \alpha$ is the one generated by the vector field $X$.

Note that the 2-form in $\mathbb{R}^{2}$ defined by $\omega=d x \wedge d y$ is symplectic, and the relation between Equations (12) and (14) is then

$$
i(X) \omega=\alpha .
$$

This means that the vector field $X$ is locally Hamiltonian, i.e., the 1-form $\alpha$ is closed if $\operatorname{div} X=0$ with respect to $\omega$. Recall that the vector field $X$ is said to be Hamiltonian when $\alpha$ is exact, see Section 5. The local expression of the divergence of the vector field $X$ given by Equation (12) with respect to the 2 -form $\omega=d x \wedge d y$ is $\operatorname{div} X=\partial P / \partial x+\partial Q / \partial y$.

Definition 2. A non-constant function $I: U \subset \mathbb{R}^{2} \rightarrow \mathbb{R}$ is called the first-integral of the system in Equation (11) or of the corresponding vector field $X$ on the open set $U$ of $\mathbb{R}^{2}$ when it remains constant along all the integral curves $(x(t), y(t))$ of $X$ contained in $U$, i.e., along the curves in $U$ that are solutions of the system.

Note that a non-constant function $I: U \subset \mathbb{R}^{2} \rightarrow \mathbb{R}$ is a first-integral on $U$ of a vector field $X$ if and only if $X_{\mid U}(I)=0$.

The remarkable point is that when we know a first-integral $I: U \rightarrow \mathbb{R}$ of Equation (11) such that $\partial I / \partial y \neq 0$ on an open subset $U$ of $\mathbb{R}^{2}$, then the condition $I(x, y)=k$ allows us to locally express the variable $y$ as a function of $x$ for each value of $k \in \mathbb{R}$, which provides us with the general solution of the Equation (13).

Recall that an integrating factor for a 1 -form $\alpha$ is a function $R$ such that $R \alpha$ is exact. Notice that as $R i(X) \omega=i(R X) \omega, R$ is an integrating factor for the 1 -form $\alpha=i(X) \omega$ associated to the vector field $X$ if and only if $R$ is a Jacobi multiplier for $X$ with respect to the volume form $\omega$.

There is an important relation between first-integrals for the vector field in Equation (12) and integrating factors for the 1-form (14) (see, e.g., Ch. X of the classical book in mechanics [1]):

Theorem 1. The function $G(x, y)$ is a first-integral for a vector field (Equation (12)) with $P Q, a$ nonvanishing function, if and only if there exists an integrating factor $R(x, y)$ for the 1-form $\alpha$ given by Equation (14) such that $R \alpha=d G$.

Proof. In fact, if $G$ is a first-integral for Equation (12), then

$$
X(G)=\frac{\partial G}{\partial x} P+\frac{\partial G}{\partial y} Q=0,
$$

and, therefore, as $Q P \neq 0$, we can define the function $R$ by the quotient

$$
R=\frac{G_{y}}{P}=-\frac{G_{x}}{Q}
$$

from here, we see that $R \alpha=G_{x} d x+G_{y} d y=d G$. 
Conversely, if $R$ is an integrating factor for Equation (14) such that $R \alpha=d G$, then, taking into account Equation (15), it trivially follows that $G_{x}=-R Q$ and $G_{y}=R P$, and therefore,

$$
X(G)=P G_{x}+Q G_{y}=0 .
$$

In more geometric terms, as $X(F)=i(X) d F$, for all functions $F \in C^{\infty}(M)$, the function $F$ is a first-integral of the motion for $X$ if and only if the 1-dimensional distribution defined by $\operatorname{Ker}(d F)$ coincides with the one generated by the vector field $X$. Note also that, in this case, as the 1-form $i(X) \omega$, where $\omega=d x \wedge d y$, also defines such a 1-dimensional distribution, there should be a function $R$ such that $R i(X) \omega=d F$, and then, such a function $R$ is an integrating factor for the 1 -form $i(X) \omega$, i.e., $R$ is a Jacobi multiplier for $X$.

There is an important application of these results to the inverse problem of the mechanics of 1-dimensional dynamical systems, namely, the fact that a single second-order ordinary differential equation, either in the autonomous or in the non-autonomous cases, can be obtained as a Euler-Lagrange equation of a function $L$ if and only if there exists a Jacobi multiplier for the associated vector field, as explained in Section 3 for autonomous systems and in Section 6 for nonautonomous ones.

\section{Jacobi Multipliers for Autonomous Second-Order Differential Equations and Lagrangians}

An autonomous second-order differential Equation (SODE), $\ddot{q}=F(q, \dot{q})$, representing a one-dimensional mechanical system has associated a system of first-order differential equations:

$$
\left\{\begin{array}{l}
\dot{q}=v \\
\dot{v}=F(q, v)
\end{array}\right.
$$

whose solutions are the integral curves of the vector field in the tangent bundle $T \mathbb{R} \equiv \mathbb{R}^{2}$.

$$
\Gamma=v \frac{\partial}{\partial q}+F(q, v) \frac{\partial}{\partial v} .
$$

As above, $\mathbb{R}^{2}$ is endowed with the symplectic form $\omega=d q \wedge d v$ and the 1-form corresponding to such a field is $\alpha_{\Gamma}=i(\Gamma) \omega=v d v-F(q, v) d q$. Now, the first-integrals of the vector field $\Gamma$ are but the constants of the motion, and the result of Theorem 1 can be reformulated in this particular case as follows:

Theorem 2. The function $G(q, v)$ is a constant of the motion for $\Gamma$ if and only if there exists a Jacobi multiplier $R$ such that $R \alpha_{\Gamma}=d G$. In this case, the multiplier given by Equation (16) is

$$
R=\frac{1}{v} \frac{\partial G}{\partial v}=-\frac{1}{F} \frac{\partial G}{\partial q} .
$$

The conditions in Equations ( 7$)$ or (9) for $R(q, v)$ to be a Jacobi multiplier for the given vector field $\Gamma$ can be put into another form. According to Equation (9), the positive function $R$ is a Jacobi multiplier if and only if

$$
\frac{\partial}{\partial q}(R v)+\frac{\partial}{\partial v}(R F)=0,
$$

i.e., more explicitly, $R$ must be such that

$$
v \frac{\partial R}{\partial q}+\frac{\partial R}{\partial v} F+R \frac{\partial F}{\partial v}=0,
$$


which taking into account that

$$
\frac{d R}{d t}=\Gamma(R)=v \frac{\partial R}{\partial q}+F \frac{\partial R}{\partial v}
$$

it can be written as

$$
\frac{d R}{d t}+R \frac{\partial F}{\partial v}=\Gamma(R)+R \frac{\partial F}{\partial v}=0
$$

or in another form,

$$
\frac{d \log R}{d t}+\frac{\partial F}{\partial v}=\Gamma(\log (R))+\frac{\partial F}{\partial v}=0 .
$$

Alternatively, it is clear that $\operatorname{div} \Gamma=\partial F / \partial v$, and then Equation (10) reduces to Equation (21).

Here, one clearly sees that if the force is velocity-independent, $F(q)$, then the system in Equation (17) has associated a divergence-free vector field $\Gamma$, and consequently, its Jacobi multipliers are the first-integrals.

In the search for a quantum model of the motion, the knowledge of a Lagrangian formulation of the classical motion may be very useful. The main result of the inverse problem of one-dimensional mechanics in this concern is:

Theorem 3. The normal form $\ddot{q}=F(q, \dot{q})$ of the differential equation determining the solutions of the Euler-Lagrange equation defined by the regular Lagrangian function $L(q, v)$ admits the function as a Jacobi multiplier:

$$
R=\frac{\partial^{2} L}{\partial v^{2}} .
$$

Conversely, if $R(q, v)$ is a Jacobi multiplier function for a second-order differential equation in normal form $\ddot{q}=F(q, \dot{q})$, then there exists a Lagrangian $L(q, v)$ for the system related to $R(q, v)$ by Equation (23). More specifically, such a Lagrangian $L$ is uniquely defined up to the addition of a gauge term by

$$
L(q, v)=\int_{v_{0}}^{v}(v-\zeta) R(q, \zeta) d \zeta+\int_{q_{0}}^{q}(R F)\left(\zeta, v_{0}\right) d \zeta,
$$

whatever values $v_{0}$ of the velocity and $q_{0}$ of the configuration be used.

Proof. In fact, if there exists a Lagrangian function $L$, the function $F$ is given by

$$
F(q, v)=\frac{1}{R}\left(\frac{\partial L}{\partial q}-v \frac{\partial^{2} L}{\partial q \partial v}\right)
$$

where the function $R$ is given by Equation (23). We can now see that, for such a function $R$, the condition of Equation (20) turns out to be identically satisfied because

$$
v \frac{\partial R}{\partial q}+\frac{\partial}{\partial v}\left(\frac{\partial L}{\partial q}-v \frac{\partial^{2} L}{\partial q \partial v}\right)=v \frac{\partial^{3} L}{\partial v^{2} \partial q}+\frac{\partial^{2} L}{\partial q \partial v}-\frac{\partial^{2} L}{\partial q \partial v}-v \frac{\partial^{3} L}{\partial v^{2} \partial q}=0,
$$

and then, $R$ is a Jacobi last multiplier.

Conversely, let $\ddot{q}=F(q, \dot{q})$ be the given second-order equation, and let $R$ be a Jacobi multiplier, i.e., a nonvanishing function satisfying Equation (20). Then, there exists a function $L$ whose Euler-Lagrange equation is equivalent to the given equation. In fact, let $L$ be a function such that the condition in Equation (23) be satisfied, i.e., the function $L$ is such that

$$
\frac{\partial L}{\partial v}=\int_{v_{1}}^{v} R(q, \zeta) d \zeta+\phi_{1}(q)
$$

and then,

$$
L(q, v)=\int_{v_{0}}^{v} d v^{\prime} \int_{v_{1}}^{v^{\prime}} R(q, \zeta) d \zeta+\phi_{1}(q) v+\phi_{0}(q)
$$


where the lower limits $v_{0}$ and $v_{1}$ are fixed but otherwise arbitrary, maybe $v_{0}=v_{1}$.

The previous formula for $L$ can be given an alternative form. First of all, by means of an integration of parts, the double integral can be written, taking $v_{1}=v_{0}$, as

$$
\int_{v_{0}}^{v}\left[\int_{v_{0}}^{v^{\prime}} R(q, \zeta) d \zeta\right] d v^{\prime}=v \int_{v_{0}}^{v} R(q, \zeta) d \zeta-\int_{v_{0}}^{v} v^{\prime} R\left(q, v^{\prime}\right) d v^{\prime}=\int_{v_{0}}^{v}(v-\zeta) R(q, \zeta) d \zeta,
$$

so that from Equation (26),

$$
L(q, v)=\int_{v_{0}}^{v}(v-\zeta) R(q, \zeta) d \zeta+\phi_{1}(q) v+\phi_{0}(q) .
$$

It is worth noting that the functions $\phi_{1}$ and $\phi_{0}$ appearing in this expression for $L$ are not completely arbitrary because they must be fixed in such a way that the Euler-Lagrange equation with such Lagrangian $L$ must be equivalent to the given $\operatorname{SODE} \ddot{q}=F(q, \dot{q})$. The term $\phi_{1}(q) v$ is a gauge term so that it can be forgotten, i.e., we can choose $\phi_{1} \equiv 0$, and then $L$ would be written, up to the addition of a gauge term, as

$$
L(q, v)=\int_{v_{0}}^{v}(v-\zeta) R(q, \zeta) d \zeta+\phi_{0}(q),
$$

where the function $\phi_{0}(q)$ is almost uniquely determined by demanding that $L$ be a Lagrangian for the given SODE, that is to say, as

$$
\frac{\partial L}{\partial v}=\int_{v_{0}}^{v} R(q, \zeta) d \zeta \text { and } \frac{\partial L}{\partial q}=\int_{v_{0}}^{v}(v-\zeta) \frac{\partial R}{\partial q}(q, \zeta) d \zeta+\phi_{0}^{\prime}(q),
$$

we have that, along the motion,

$$
0=\frac{d}{d t}\left(\frac{\partial L}{\partial v}\right)-\frac{\partial L}{\partial q}=R(q, v) F(q, v)+\int_{v_{0}}^{v} \zeta \frac{\partial R}{\partial q}(q, \zeta) d \zeta-\phi_{0}^{\prime}(q) .
$$

However, as $R$ is assumed to be a Jacobi multiplier, Equation (20) holds, i.e.,

$$
\frac{\partial(R v)}{\partial q}(q, \zeta)=-\frac{\partial(R F)}{\partial v}(q, \zeta)
$$

and we can write Equation (29) as follows:

$$
R F(q, v)-\int_{v_{0}}^{v} \frac{\partial(R F)}{\partial v}(q, \zeta) d \zeta-\phi_{0}^{\prime}(q)=0,
$$

from where we arrive at $R F\left(q, v_{0}\right)-\phi_{0}^{\prime}(q)=0$, i.e., Euler-Lagrange equation is equivalent to $\phi_{0}^{\prime}(q)=(R F)\left(q, v_{0}\right)$, no matter of the value $v_{0}$ of the velocity $v$, from where we obtain

$$
\phi_{0}(q)=\int_{q_{0}}^{q}(R F)\left(\zeta, v_{0}\right) d \zeta .
$$

The expression in Equation (28) for the Lagrangian determined by the Jacobi multiplier $R$ reduces to Equation (24) for this value of the function $\phi_{0}$. Therefore, we can find a Lagrangian by means of two quadratures.

The final expression in Equation (24) for the Lagrangian and its extension to the time-dependent case are due to Yan [40] (see also [36,41]). 
Corollary 1. If a system with one degree of freedom admits two different regular Lagrangians $L_{1}$ and $L_{2}$, then the function $f$ defined by

$$
f \frac{\partial^{2} L_{1}}{\partial v^{2}}=\frac{\partial^{2} L_{2}}{\partial v^{2}}
$$

is a constant of the motion. Conversely, if $f$ is a constant of the motion and $L_{1}$ is a Lagrangian for the second-order differential equation, then

$$
R=f \frac{\partial^{2} L_{1}}{\partial v^{2}}
$$

is a Jacobi multiplier, and there exists another Lagrangian function $L_{2}$ such that Equation (31) holds.

Proof. If the function $f$ is the quotient of two different Jacobi multipliers, $f$ is a first-integral, and conversely if $f$ is a first-integral and $L_{1}$ is a Lagrangian, then $R$ given by the above expression is also a Jacobi multiplier.

The result of this corollary is usually attributed in the physics literature to Currie and Saletan [6] but actually dates back to Jacobi's time.

\section{Applications to Some SODE's and Associated Lagrangians}

A particular example of the SODE equation was recently discussed in [26,42], where the following type of Liénard differential equations [43] is studied:

$$
\ddot{x}+f(x) \dot{x}+g(x)=0,
$$

with an associated system

$$
\left\{\begin{array}{l}
\dot{x}=v \\
\dot{v}=-f(x) v-g(x)
\end{array},\right.
$$

i.e., $F(x, v)=-f(x) v-g(x)$.

If we try to determine a Jacobi multiplier of the form $R(x, v)=u(x, v)^{\beta}$ with $u$, which is a function of the form $u(x, v)=v+\varphi(x)$ and $\beta \in \mathbb{R}$, then the condition in Equation (22) for the Jacobi multiplier becomes

$$
\beta \frac{\dot{u}}{u}-f(x)=0,
$$

and taking into account the assumed form for the function $u$ and making use of the equation of motion (Equation (32)), we obtain

$$
\beta\left[-f(x) v-g(x)+\varphi^{\prime}(x) v\right]-f(x)(v+\varphi(x))=0,
$$

and therefore,

$$
-\beta g(x)=f(x) \varphi(x), \quad(\beta+1) f(x)=\beta \varphi^{\prime}(x) .
$$

The first equation implies that the function $\varphi(x)$ must be $\varphi(x)=-\beta g(x) / f(x)$, and then, the second equation implies the necessary condition for the existence of such a solution, usually called the Chiellini condition [44]:

$$
-\beta^{2} \frac{d}{d x}\left(\frac{g(x)}{f(x)}\right)=(\beta+1) f(x),
$$

and consequently, if Equation (34) is satisfied, the function

$$
R(x, v)=\left(v-\beta \frac{g(x)}{f(x)}\right)^{\beta}
$$


is a Jacobi multiplier for Equation [26,42].

The Lagrangian describing the system can easily be obtained by computing the integrals in the general formula in Equation (24). An integration of parts in the first integral in Equation (24), with $v_{0}=0$, yields

$$
\begin{aligned}
\int_{0}^{v}(v-\zeta)\left(\zeta-\beta \frac{g(x)}{f(x)}\right)^{\beta} d \zeta & =\frac{1}{(\beta+1)(\beta+2)}\left(v-\beta \frac{g(x)}{f(x)}\right)^{\beta+2} \\
& -\frac{1}{(\beta+1)(\beta+2)}\left(-\beta \frac{g(x)}{f(x)}\right)^{\beta+2}
\end{aligned}
$$

up to the addition of a gauge term. On the other hand, by using Chiellini's condition (34), the second integral is

$$
\int_{x_{0}}^{x} R(\zeta, 0) F(\zeta, 0) d \zeta=\frac{(-\beta)^{\beta+2}}{(\beta+1)(\beta+2)}\left(\frac{g(x)}{f(x)}\right)^{\beta+2}+\text { const }
$$

Finally, the Lagrangian for Equation (32) associated to the multiplier is just

$$
L(x, v)=\frac{1}{(\beta+1)(\beta+2)}\left(v-\beta \frac{g(x)}{f(x)}\right)^{\beta+2} .
$$

For instance, consider the following equation [26,45-47]:

$$
\ddot{x}+k x \dot{x}+\frac{k^{2}}{9} x^{3}+\lambda x=0,
$$

which corresponds to $f(x)=k x$ and $g(x)=\frac{k^{2}}{9} x^{3}+\lambda x$, and therefore,

$$
\frac{d}{d x}\left(\frac{g(x)}{f(x)}\right)=\frac{d}{d x}\left(\frac{k}{9} x^{2}+\frac{\lambda}{k}\right)=\frac{2}{9} k x=\frac{2}{9} f .
$$

There are two values of $\beta$ such that $(\beta+1) / \beta^{2}=-2 / 9$, i.e.,

$$
2 \beta^{2}+9 \beta+9=0 \text {, }
$$

which are given by $\beta_{1}=-3$ and $\beta_{2}=-3 / 2$. For $\beta=-3$, we obtain $\varphi(x)=\frac{k}{3} x^{2}+\frac{3}{k} \lambda$, while for $\beta=-3 / 2$, we get $\varphi(x)=\frac{k}{6} x^{2}+\frac{3}{2 k} \lambda$. Consequently, two Jacobi multipliers for the system are

$$
R_{1}(x, v)=\left[v+3\left(\frac{k}{9} x^{2}+\frac{\lambda}{k}\right)\right]^{-3}, \quad R_{2}(x, v)=\left[v+\frac{3}{2}\left(\frac{k}{9} x^{2}+\frac{\lambda}{k}\right)\right]^{-3 / 2} .
$$

The corresponding Lagrangians (Equation (35)) are given up to a gauge term by:

$$
L_{1}=\frac{1}{2} \frac{1}{v+\frac{k}{3} x^{2}+\frac{3}{k} \lambda} \quad \text { and } \quad L_{2}=-4 \sqrt{v+\frac{k}{6} x^{2}+\frac{3}{2 k} \lambda} .
$$

As indicated in the more general case, the function $\phi_{0}$ in both cases is constant.

A particular example is the case $\lambda=0$ [48], and when $k=3$, we find the abovementioned second-order element of the Riccati chain (see, e.g., $[13,21,26])$

$$
\ddot{x}+3 x \dot{x}+x^{3}=0,
$$


which, as indicated before, admits two Jacobi multipliers given by

$$
R_{1}(x, v)=\left(v+x^{2}\right)^{-3}, \quad R_{2}(x, v)=\left(v+\frac{1}{2} x^{2}\right)^{-3 / 2},
$$

and, up to a constant factor, the corresponding Lagrangians (Equation (35)) are

$$
L_{1}=\frac{1}{v+x^{2}}, \quad L_{2}=\sqrt{v+\frac{1}{2} x^{2}} .
$$

As far as the important application of Jacobi multipliers to the inverse problem of the mechanics of one-dimensional systems we are interested in, a remarkable result is the following:

Theorem 4. If $G(q, v)$ is a constant of the motion for the autonomous second-order differential equation vector field (Equation (18)), then there is a regular Lagrangian for the associated vector field given (see [34-37]) up to a gauge term by

$$
L(q, v)=v \int_{v_{0}}^{v} \frac{G(q, \zeta)}{\zeta^{2}} d \zeta
$$

Proof. In fact, as $G(q, v)$ is a constant of the motion, there exists a Jacobi multiplier $R$ given by Equation (19). The preceding Theorem 2 shows us that the related Lagrangian $L$ is given by Equation (24), with $R$ related to $G$ by Equation (19), and then

$$
L(q, v)=\int_{v_{0}}^{v} \frac{v-\zeta}{\zeta} \frac{\partial G}{\partial v}(q, \zeta) d \zeta-\int_{q_{0}}^{q} \frac{\partial G}{\partial q}\left(\zeta, v_{0}\right) d \zeta .
$$

After an integration of parts in the first of the integrals, we immediately obtain the Lagrangian in Equation (36) for the given vector field. Moreover, as

$$
\frac{\partial L}{\partial v}=\int_{v_{0}}^{v} \frac{G(q, \zeta)}{\zeta^{2}} d \zeta+\frac{G(q, v)}{v},
$$

the energy $E=v(\partial L / \partial v)-L$ is just the constant of the motion $G(q, v)$ because

$$
v \frac{\partial L}{\partial v}-L=v \int_{v_{0}}^{v} \frac{G(q, \zeta)}{\zeta^{2}} d \zeta+G-v \int_{v_{0}}^{v} \frac{G(q, \zeta)}{\zeta^{2}} d \zeta=G .
$$

A trivial example is the free particle, for which $G=v^{2}$ and $L=v^{2}$.

Notice that the above result from Equation (36) also means that, in the autonomous case, as according to Theorem 2, the knowledge of one constant of the motion determines a Jacobi multiplier, and then as a result of Theorem 3, we also have a Lagrangian description of the dynamics. The result of this theorem can be useful when one can find a first-integral for the given second-order differential equation vector field. As a first instance, when the function $F(q, v)$ appearing in the expression of the SODE vector field $\Gamma$ is factorisable, i.e., of the form $F(q, v)=f(q) h(v)$, then a first-integral $I(q, v)$ is a solution of the partial differential equation

$$
v \frac{\partial I}{\partial q}+f(q) h(v) \frac{\partial I}{\partial v}=0,
$$

with an associated system

$$
\frac{d q}{v}=\frac{d v}{f(q) h(v)}
$$

i.e.,

$$
f(q) d q=\frac{v d v}{h(v)}
$$


from which we have the first-integral

$$
I(q, v)=\int^{v} \frac{\zeta}{h(\zeta)} d \zeta-\int^{q} f(\zeta) d \zeta
$$

For instance, the Mathews-Lakshmanan oscillator [49-52]

$$
\ddot{q}=F(q, \dot{q})=\frac{-a+\lambda \dot{q}^{2}}{1+\lambda q^{2}} q, \quad a, \lambda \in \mathbb{R},
$$

is the particular instance corresponding to

$$
f(q)=\frac{q}{1+\lambda q^{2}}, \quad h(v)=-a+\lambda v^{2},
$$

and we obtain a first-integral

$$
I(q, v)=\int_{v_{0}}^{v} \frac{\zeta}{-a+\lambda \zeta^{2}} d \zeta-\int^{q} \frac{\zeta}{1+\lambda \zeta^{2}} d \zeta=\frac{1}{2 \lambda}\left(\log \left(-a+\lambda v^{2}\right)-\log \left(1+\lambda q^{2}\right)\right)
$$

and, therefore, any first-integral is a function of

$$
I(q, v)=\frac{\lambda v^{2}-a}{1+\lambda q^{2}}
$$

Remark that Equation (36) allows us to write the corresponding Lagrangian as a real multiple of

$$
L(q, v)=v \int_{0}^{v} \frac{\lambda \zeta^{2}-a}{1+\lambda q^{2}} \frac{1}{\zeta^{2}} d \zeta=\frac{\lambda v^{2}+a}{1+\lambda q^{2}}
$$

which shows that $I$ is the energy corresponding to this Lagrangian. Moreover, the usual Lagrangian for Equation (37) is

$$
L^{\prime}(q, v)=\frac{1}{2} \frac{v^{2}-a q^{2}}{1+\lambda q^{2}}
$$

which satisfies $L-2 \lambda L^{\prime}=a$.

Another example studied in [27] is the homogeneous differential equation:

$$
\ddot{x}=\dot{x} g\left(\frac{\dot{x}}{x}\right),
$$

i.e., the function $F$ in Equation (18) is given by

$$
F(x, v)=v g\left(\frac{v}{x}\right)
$$

and the SODE vector field by

$$
\Gamma(x, v)=v \frac{\partial}{\partial x}+v g\left(\frac{v}{x}\right) \frac{\partial}{\partial v} .
$$

A constant of motion $I(x, v)$ is a solution of the partial differential equation

$$
v \frac{\partial I}{\partial x}+v g\left(\frac{v}{x}\right) \frac{\partial I}{\partial v}=0
$$

with the associated system

$$
\frac{d x}{v}=\frac{d v}{v g(v / x)}
$$


and, therefore, if in the first-order differential equation

$$
\frac{d v}{d x}=g\left(\frac{v}{x}\right)
$$

we change the variable $v$ by $z=v / x$, and the preceding equation transforms into

$$
\frac{d z}{d x}=\frac{g(z)-z}{x},
$$

and we see that the constant of motion I must be a function of

$$
I(x, v)=\log x-\int^{v / x} \frac{d \zeta}{g(\zeta)-\zeta} .
$$

The particular example analysed in [27] corresponds to

$$
F(x, v)=2 v \sqrt{\frac{v}{x}}+\frac{v^{2}}{x} \Longleftrightarrow g(\zeta)=2 \sqrt{\zeta}+\zeta,
$$

from which we obtain

$$
I(x, v)=\log x-\int^{v / x} \frac{d \zeta}{2 \sqrt{\zeta}}=\log x-\sqrt{\frac{v}{x}} .
$$

The Lagrangian corresponding to such a constant of motion is given by

$$
L(x, v)=v \int^{v} \frac{\log x-\sqrt{\frac{\zeta}{x}}}{\zeta^{2}} d \zeta=-\log x+2 \sqrt{\frac{v}{s} \cdot x},
$$

up to a gauge term.

Once the function $L$ has been found through the Jacobi multiplier $R$, we can pass to the Hamiltonian formulation by means of the Legendre transformation. Note, however, that the Hamiltonian $H$ depends on the choice of the gauge ambiguity for the Lagrangian.

The method developed in [27] for the search of a Hamiltonian formulation of a given SODE constructed by a constant of motion immediately follows from the Lagrangian Equation (36). Actually, the generalised momentum is

$$
p=\frac{\partial L}{\partial v}=\int_{v_{0}}^{v} \frac{G(q, \zeta)}{\zeta^{2}} d \zeta+\frac{G(q, v)}{v}=\int_{v_{0}}^{v} \frac{1}{\zeta} \frac{\partial G}{\partial v}(q, \zeta) d \zeta+\frac{G\left(q, v_{0}\right)}{v_{0}},
$$

where an integration by parts has been carried out.

This result is to be compared with Equation (2.5) in [27]. Our result includes a function $\varphi(q)$ uniquely determined by the constant of motion $G$ itself. Such a function reappears when we change $L$ to the gauge equivalent Lagrangian $L^{\prime}=L+\varphi(q) v$. The Hamiltonian function $H=p v-L$ is

$$
H(q, p)=G(q, v(q, p)),
$$

from which the corresponding Hamilton equations can be obtained.

Consider, as an example, the previous example. The Legendre transformation can be carried out, giving rise to

$$
p=\frac{1}{\sqrt{x v}}
$$

while the Hamiltonian is

$$
H(x, p)=I\left(x, \frac{1}{x p^{2}}\right)=\log x-\frac{1}{x p},
$$


from where the corresponding Hamilton equations can be obtained. The original secondorder differential equation can also be recovered.

\section{A Quick Geometric Presentation of Hamiltonian and Lagrangian Frameworks}

We recall that $(M, \omega)$ is a symplectic manifold if $M$ is a finite-dimensional differentiable manifold and $\omega$ is a nondegenerate 2-form, which satisfies $d \omega=0$ (that is, $\omega \in Z^{2}(M)$ ). The dimension of $M$ is necessarily even, $\operatorname{dim} M=2 n$. For general results, reference textbooks are, for instance, [9,11,12]. By nondegeneracy of $\omega$, we mean that for every point $u \in M$ the map $\widehat{\omega}_{u}: T_{u} M \rightarrow T_{u}^{*} M$, given by: $\left\langle\widehat{\omega}_{u}(v), v^{\prime}\right\rangle=\omega_{u}\left(v, v^{\prime}\right)$ with $v, v^{\prime} \in T_{u} M$, has a maximal rank, i.e., $\omega^{\wedge n} \neq 0$. Such a map $\widehat{\omega}$ is a base-preserving fibred map, and hence, it induces a mapping between the linear spaces of sections which, with a slight abuse of notation, we will also write $\widehat{\omega}: \mathfrak{X}(M) \rightarrow \Lambda^{1}(M)$. All these symplectic manifolds are locally similar because Darboux proved that for each point $u \in M$, there is a local chart $(U, \phi)$ such that if $\phi=\left(q^{1}, \ldots, q^{n} ; p_{1}, \ldots, p_{n}\right)$, then $\left.\omega\right|_{u}=d q^{i} \wedge d p_{i}$. Such coordinates are said to be Darboux coordinates.

As closed, and in particular, exact, 1-forms are distinguished elements in $\Lambda^{1}(M)$, the corresponding vector fields are called locally Hamiltonian vector fields and Hamiltonian vector fields, respectively. If $H \in C^{\infty}(M)$, the Hamiltonian vector field $X_{H}$ associated with the Hamiltonian $H$ is the unique vector field that satisfies $\widehat{\omega}\left(X_{H}\right)=i\left(X_{H}\right) \omega=d H$. The set of Hamiltonian vector fields will be denoted $\mathfrak{X}_{\mathrm{H}}(M, \omega)$ and that of locally Hamiltonian vector fields $\mathfrak{X}_{\mathrm{LH}}(M, \omega)$, i.e., $\mathfrak{X}_{\mathrm{LH}}(M, \omega)=\widehat{\omega}^{-1}\left(Z^{1}(M)\right)$ and $\mathfrak{X}_{\mathrm{H}}(M, \omega)=\widehat{\omega}^{-1}\left(B^{1}(M)\right)$. Observe that $\widehat{\omega}^{-1}$ is an isomorphism of real linear spaces. The Cartan homotopy identity, i.e., $\mathcal{L}_{X} \alpha=i(X) d \alpha+d[i(X) \alpha]$, for any $\alpha \in \wedge^{p}(M)$, shows that $\mathcal{L}_{X_{H}} \omega=0$, and, moreover, $X \in \mathfrak{X}(M)$, is such that $\mathcal{L}_{X} \omega=0$ if and only if $i(X) \omega$ is a closed 1-form, i.e., $X \in$ $\mathfrak{X}_{\mathrm{LH}}(M, \omega)$. In Darboux coordinates, the Hamiltonian vector field $X_{H}$ corresponding to the function $H$ is given by

$$
X_{H}=\frac{\partial H}{\partial p_{i}} \frac{\partial}{\partial q^{i}}-\frac{\partial H}{\partial q^{i}} \frac{\partial}{\partial p_{i}},
$$

and therefore, the equations determining its integral curves are similar to Hamilton equations. We can define the Poisson bracket of two functions $F, G \in C^{\infty}(M)$ in a $2 n$-dimensional symplectic manifold $(M, \omega)$ as being the function $\{F, G\}$ given by:

$$
\{F, G\}=\omega\left(X_{F}, X_{G}\right)=d F\left(X_{G}\right)=-d G\left(X_{F}\right),
$$

which in Darboux coordinates, for $\omega$, looks like the usual one:

$$
\{F, G\}=\frac{\partial F}{\partial q^{i}} \frac{\partial G}{\partial p_{i}}-\frac{\partial F}{\partial p_{i}} \frac{\partial G}{\partial q^{i}} .
$$

Remark that if $X, Y \in \mathfrak{X}_{\mathrm{LH}}(M, \omega)$, the commutator $[X, Y]$ is a Hamiltonian vector field, with Hamiltonian $\omega(Y, X)$, because from the relation $i(X) \mathcal{L}_{Y} \alpha-\mathcal{L}_{Y} i(X) \alpha=i([X, Y]) \alpha$, valid for any form $\alpha$, we obtain:

$$
i([X, Y]) \omega=i(X) \mathcal{L}_{Y} \omega-\mathcal{L}_{Y} i(X) \omega=-\mathcal{L}_{Y} i(X) \omega,
$$

and, therefore,

$$
i([X, Y]) \omega=-i(Y) d[i(X) \omega]-d[i(Y) i(X) \omega]=-d[\omega(X, Y)] .
$$

Consequently, the set $\mathfrak{X}_{\mathrm{LH}}(M, \omega)$ is a Lie algebra, and $\mathfrak{X}_{\mathrm{H}}(M, \omega)$ is an ideal in $\mathfrak{X}_{\mathrm{LH}}(M, \omega)$. The preceding property means that if $F, G \in C^{\infty}(M)$, then we have that $d\{F, G\}=-i\left(\left[X_{F}, X_{G}\right]\right) \omega$, i.e., $\left[X_{F}, X_{G}\right]=X_{\{G, F\}}$. 
This Poisson bracket $\{\cdot, \cdot\}$ is a skewsymmetric $\mathbb{R}$-bilinear map on $C^{\infty}(M)$ such that it satisfies Jacobi's identity as a consequence of $\omega$ being closed. In fact, if $F, G, H \in C^{\infty}(M)$,

$$
\begin{aligned}
(d \omega)\left(X_{F}, X_{G}, X_{H}\right) & =X_{F} \omega\left(X_{G}, X_{H}\right)-X_{G} \omega\left(X_{F}, X_{H}\right)+X_{H} \omega\left(X_{F}, X_{G}\right) \\
& -\omega\left(\left[X_{F}, X_{G}\right], X_{H}\right)+\omega\left(\left[X_{F}, X_{H}\right], X_{G}\right)-\omega\left(\left[X_{G}, X_{H}\right], X_{F}\right),
\end{aligned}
$$

and taking into account that $X_{F} \omega\left(X_{G}, X_{H}\right)=X_{F}\{G, H\}=\{\{G, H\}, F\}$ and that $\omega\left(\left[X_{F}, X_{G}\right], X_{H}\right)$, using that $\left[X_{F}, X_{G}\right]=X_{\{G, F\}}$, it can also be rewritten in the form $\omega\left(\left[X_{F}, X_{G}\right], X_{H}\right)=\omega\left(X_{\{G, F\}}, X_{H}\right)=\{\{G, F\}, H\}$, as well as the corresponding expressions for each cyclic reordering, we find that

$$
(d \omega)\left(X_{F}, X_{G}, X_{H}\right)=2[\{\{G, H\}, F\}+\{\{H, F\}, G\}+\{\{F, G\}, H\}]=0 .
$$

Recall that the values of Hamiltonian vector fields in each point span the tangent space in the point, and therefore, the Jacobi identity holds if and only if $\omega$ is closed. The two main examples of Hamiltonian dynamical systems are those of Hamiltonian systems in the cotangent bundle $T^{*} Q$ of a manifold $Q$ and those defined by regular Lagrangians in the tangent bundle $T Q$. In fact, the cotangent bundle $\pi: T^{*} Q \rightarrow Q$ is endowed with a canonical 1-form $\theta \in \Lambda^{1}\left(T^{*} Q\right)$ defined by $\theta_{\alpha}=\pi_{\alpha}^{*} \alpha$. Then, $\omega=-d \theta$ is a canonical symplectic form on $T^{*} Q$. The geometric framework for the study of Lagrangian mechanics is that of tangent bundles [4,10,53]. The tangent bundle $\tau: T Q \rightarrow Q$ is characterised by two geometric tensors: the vertical endomorphism $S$, a (1,1)-tensor field on $T Q$, also called tangent structure, which satisfies $\operatorname{Im} S=\operatorname{Ker} S$; and an integrability condition, where its Nijenhuis tensor $N_{S}$ is null, and the Liouville vector field $\Delta$ generates dilations along fibres in $T Q$ [54].

If $(U, \varphi)$ is a local chart on $Q$ and $\mathrm{pr}^{i}: \mathbb{R}^{n} \rightarrow \mathbb{R}$ are the natural projections on the $i$-th-factor and $q^{i}=\operatorname{pr}^{i} \circ \varphi$, we define the coordinate system $\left(U, q^{1}, \ldots, q^{n}\right)$ on $Q$, and the corresponding chart in $\mathcal{U}=\tau^{-1}(\mathcal{U})$ given by $\left(\mathcal{U}, \varphi, \varphi_{*}\right)$, defines a coordinate system $\left(U, q^{1}, \ldots, q^{n}, v^{1}, \ldots, v^{n}\right)$ on $T Q$. Correspondingly, we consider the coordinate basis of $\mathfrak{X}(U)$, usually denoted $\left\{\partial / \partial q^{j} \mid j=1, \ldots, n\right\}$, and its dual basis for $\wedge^{1}(U)$, $\left\{d q^{j} \mid j=1, \ldots, n\right\}$. Then, a vector $v$ in a point $q \in U$ is $v=v^{j}\left(\partial / \partial q^{j}\right)_{\mid q}$, i.e., $v^{i}=d q^{i}(v)$, while a covector $\alpha$ in a point $q \in U$ is $\alpha=p_{j}\left(d q^{j}\right)_{\mid q}$, with $p_{i}=\alpha\left(\partial / \partial q^{i}\right)_{\mid q}$. With this notation, the coordinate expressions of the vertical endomorphism $S$ and the Liouville vector field $\Delta$ are $[4,10]$ :

$$
S(q, v)=\frac{\partial}{\partial v^{i}} \otimes d q^{i}, \quad \Delta(q, v)=v^{i} \frac{\partial}{\partial v^{i}},
$$

while the coordinate expression of the 1 -form $\theta$ is

$$
\theta(q, p)=p_{i} d q^{i}
$$

which shows that $\omega=-d \theta$ is a canonical symplectic form on $T^{*} Q$.

The vector field $\Delta$ and the tensor field $S$ can be used to select a special kind of vector field whose integral curves are given by lifting solutions of second-order differential equations. These vector fields called second-order differential equation vector fields $D \in$ $\mathfrak{X}(T Q)$ (hereafter shortened as SODE vector fields) are characterised by $S(D)=\Delta$. The expression of a SODE vector field $D$ in the above-mentioned local coordinates is

$$
D(q, p)=v^{i} \frac{\partial}{\partial q^{i}}+f^{i}(q, v) \frac{\partial}{\partial v^{i}}
$$


Recall also that given a Lagrangian $L \in C^{\infty}(T Q)$, we can define a 1-form $\theta_{L}=d L \circ S$ and the exact 2-form $\omega_{L}=-d \theta_{L}$. When $\omega_{L}$ is regular the Lagrangian $L$ is said to be regular, and then the dynamics are given by the uniquely defined SODE vector field $\Gamma_{L}$ such that

$$
i\left(\Gamma_{L}\right) \omega_{L}=d E_{L} \Longleftrightarrow \mathcal{L}_{\Gamma_{L}} \theta_{L}-d L=0,
$$

where the energy function $E_{L} \in C^{\infty}(T Q)$ is defined by $E_{L}=\Delta(L)-L$. The curves in $Q$ that are projections of the integral curves of $\Gamma_{L}$ are the solutions of the system of Euler-Lagrange equations.

Remark that the condition $i\left(\Gamma_{L}\right) \omega_{L}=d E_{L}$ implies that $\mathcal{L}_{\Gamma_{L}} \omega_{L}=0$ because $\mathcal{L}_{\Gamma_{L}} \omega_{L}=$ $d\left(i\left(\Gamma_{L}\right) \omega_{L}\right)$. In usual local tangent bundle coordinates, the expressions of $\theta_{L}$ and $E_{L}$ are

$$
\theta_{L}=\frac{\partial L}{\partial v^{i}} d q^{i}, \quad E_{L}=v^{i} \frac{\partial L}{\partial v^{i}}-L,
$$

while that of $\omega_{L}$ is:

$$
\omega_{L}=\frac{\partial^{2} L}{\partial q^{j} \partial v^{i}} d q^{i} \wedge d q^{j}+\frac{\partial^{2} L}{\partial v^{k} \partial v^{j}} d q^{j} \wedge d v^{k} .
$$

We can give intrinsic proof of the direct part of Theorem 3 (see, e.g., [55]). Recall first that in the geometric approach to Lagrangian mechanics, we have summarised in Section 5 when one starts with a 1-dimensional configuration space, $Q \equiv \mathbb{R}$, and hence, $T Q \equiv \mathbb{R} \times \mathbb{R}$, and the expressions of $S$ and $\theta_{L}$ in local coordinates $(q, v)$ are

$$
S=\frac{\partial}{\partial v} \otimes d q, \quad \theta_{L}=\frac{\partial L}{\partial v} d q .
$$

Then, given a function $L \in C^{\infty}(T Q)$ as Lagrangian,

$$
\omega_{L}=\frac{\partial^{2} L}{\partial v^{2}} d q \wedge d v=\frac{\partial^{2} L}{\partial v^{2}} \omega,
$$

where $\omega$ is the symplectic form $\omega=d q \wedge d v$ in $T Q$, and then $\omega_{L}$ is a regular Lagrangian when $\partial^{2} L / \partial v^{2} \neq 0$ because $\omega_{L}$ is a symplectic form. The dynamical vector field $\Gamma_{L}$ is defined by $i\left(\Gamma_{L}\right) \omega_{L}=d E_{L}$, which implies the condition $\mathcal{L}_{\Gamma_{L}} \omega_{L}=0$, and then

$$
d\left(\frac{\partial^{2} L}{\partial v^{2}} i\left(\Gamma_{L}\right) \omega\right)=d\left(i\left(\Gamma_{L}\right) \omega_{L}\right)=0 .
$$

This shows that $R=\partial^{2} L / \partial v^{2}$ is a Jacobi multiplier for the vector field $\Gamma_{L}$ in the oriented manifold $(T Q, \omega)$.

The generalisation of this result to the $n$-dimensional case is immediate. If a SODE vector field $\Gamma$ admits a Lagrangian description by a regular function $L$, then $i(\Gamma) \omega_{L}=d E_{L}$ and, consequently, $\mathcal{L}_{\Gamma} \omega_{L}=0$, from which we see that the volume form $\left(\omega_{L}\right)^{\wedge n}$ is an invariant volume form. In a given chart $\left(q^{1}, \ldots, q^{n}, v^{1}, \ldots, v^{n}\right)$ of $T Q$ induced from one $\left(q^{1}, \ldots, q^{n}\right)$ in $Q$, we have an associated volume form $\Omega=d q^{1} \wedge \cdots \wedge d q^{n} \wedge d v^{1} \wedge \cdots \wedge d v^{n}$, and as $\left(\omega_{L}\right)^{\wedge n}$ is a constant multiple of $\operatorname{det} W \Omega$, where $W$ is the matrix with elements $W_{i j}=\partial^{2} L / \partial v^{i} \partial v^{j}$, we see that $\operatorname{det} W$ is a Jacobi multiplier for the vector field $\Gamma$ with respect to the volume form $\Omega$. 


\section{Jacobi Multipliers for Non-Autonomous Second-Order Differential Equations and Lagrangians}

The results in the preceding section can be extended and generalised to the nonautonomous case. A non-autonomous second-order differential equation $\ddot{q}=F(t, q, \dot{q})$ has associated a system of first-order differential equations:

$$
\left\{\begin{array}{l}
\dot{t}=1 \\
\dot{q}=v \\
\dot{v}=F(t, q, v)
\end{array}\right.
$$

whose solutions are the integral curves of the vector field in the manifold $\mathbb{R}^{3} \equiv \mathbb{R} \times T \mathbb{R}$

$$
\Gamma=\frac{\partial}{\partial t}+v \frac{\partial}{\partial q}+F(t, q, v) \frac{\partial}{\partial v} .
$$

The manifold $\mathbb{R}^{3}$ is supposed to be endowed with the natural volume form $\Omega=$ $d t \wedge d x \wedge d v$ and then $\operatorname{div} \Gamma=\partial F / \partial v$, and consequently, the positive function $R$ is a Jacobi multiplier for such a field with respect to $\Omega$ if and only if

$$
\Gamma(R)+R \frac{\partial F}{\partial v}=0,
$$

which can also be written as Equations (21) and (22) with the new meanings for $d R / d t$ and $\Gamma(R)$. Note that, once again, $f R$ satisfies the same equation as $R$ if $f$ is a constant of the motion.

Remark that the condition in Equation (2) for the function $g$, as mentioned in the introduction, is exactly that the function $g$ be a Jacobi multiplier. Moreover, we can state an analogous property to that of Theorem 3:

Theorem 5. The normal form $\ddot{q}=F(t, q, \dot{q})$ of the differential equation determining the solutions of the Euler-Lagrange equation, defined by a regular Lagrangian L, admits, as a Jacobi multiplier, the function

$$
R(t, q, v)=\frac{\partial^{2} L}{\partial v^{2}}
$$

Conversely, if $R(t, q, v)$ is a Jacobi multiplier for the vector field in Equation (45), then the associated SODE $\ddot{q}=F(t, q, \dot{q})$ admits a regular Lagrangian $L$ related to $R$ by Equation (47). More specifically, a regular Lagrangian $L$ for the dynamics is explicitly given in terms of $R$ by

$$
L(t, q, v)=\int_{v_{0}}^{v}(v-\zeta) R(t, q, \zeta) d \zeta+\int_{q_{0}}^{q} F\left(t, \zeta, v_{0}\right) R\left(t, \zeta, v_{0}\right) d \zeta,
$$

no matter the values of $v_{0}$ and $q_{0}$.

The proof of this statement goes along the same steps as those of Theorem 2 for the autonomous case, using the condition of Equation (46) for the multiplier instead of Equation (21) when needed.

In fact, if the given SODE is equivalent to the Euler-Lagrange equation defined by $L(t, q, v)$, then

$$
\frac{\partial^{2} L}{\partial v^{2}} F=-\frac{\partial^{2} L}{\partial q \partial v} v-\frac{\partial^{2} L}{\partial t \partial v}+\frac{\partial L}{\partial q}
$$


so that it is easy to check that $R=\partial^{2} L / \partial v^{2}$ satisfies the condition of Equation (46) because

$$
\begin{aligned}
\frac{\partial R}{\partial t} & +v \frac{\partial R}{\partial q}+\frac{\partial}{\partial v}\left(\frac{\partial L}{\partial q}-v \frac{\partial^{2} L}{\partial q \partial v}-\frac{\partial^{2} L}{\partial t \partial v}\right)= \\
& =\frac{\partial^{3} L}{\partial v^{2} \partial t}+v \frac{\partial^{3} L}{\partial v^{2} \partial q}+\frac{\partial^{2} L}{\partial q \partial v}-\frac{\partial^{2} L}{\partial q \partial v}-v \frac{\partial^{3} L}{\partial v^{2} \partial q}-\frac{\partial^{3} L}{\partial v^{2} \partial t}=0,
\end{aligned}
$$

that is to say, $R$ is a Jacobi multiplier for the vector field in Equation (45) determined by the SODE.

As far as the converse part of the Theorem is concerned, note that the multiplier $R$ and the functions $\phi_{1}$ and $\phi_{0}$ appearing in the proof of Theorem 3 are now also allowed to depend on $t$. A term, such as $\phi(t, q) v$, splits into a gauge term and a function of $(t, q)$ alone.

There is an intrinsic derivation of the direct part of Theorem 5. Recall that the geometric approach to Lagrangian formulation in a one-dimensional configuration space $Q$ is developed in the evolution space $\mathbb{R} \times T Q$ and that there is a 1-form $\theta$ in $\mathbb{R} \times T Q$, called the contact form, with local expression in a chart on $\mathbb{R} \times T Q$ induced from one chart in $Q$, given by $\theta=d q-v d t$, such that together with the 1 -fom $\Psi=d v-F d t$ are such that $\{d t, \theta, \Psi\}$ is the dual basis of the basis of vector fields $\{\Gamma, \partial / \partial q, \partial / \partial v\}$. Then, if the vector field in Equation (45) admits a Lagrangian fomulation, $\Gamma$ is determined by $i(\Gamma) \Omega_{L}=0$ and the condition $i(\Gamma) d t=1$, where

$$
\Theta_{L}=L d t+\frac{\partial L}{\partial v} \theta, \quad \Omega_{L}=-d \Theta_{L}
$$

However, as $\Omega_{L}=-d \Theta_{L}$ is an exact form, $\mathcal{L}_{\Gamma} \Omega_{L}=d\left(i(\Gamma) \Omega_{L}\right)=0$, and therefore, as $\mathcal{L}_{\Gamma}(d t)=0$, the vector field $\Gamma$ is divergence-free with respect to the volume form $d t \wedge \Omega_{L}$. Taking into account that $d t \wedge d \theta=0$, we will have that

$$
d t \wedge \Omega_{L}=-d t \wedge\left(d\left(\frac{\partial L}{\partial v}\right) \wedge \theta\right)=\frac{\partial^{2} L}{\partial v^{2}} d t \wedge d q \wedge d v
$$

from which we see that $i(\Gamma)\left(d t \wedge \Omega_{L}\right)=\Omega_{L}$ is exact, and this implies that $\partial^{2} L / \partial v^{2}$ is a Jacobi multiplier for the vector field $\Gamma$ with respect to the volume form defined in the chart $d t \wedge d q \wedge d v$.

There is a useful result for Equation (44) and its associated vector field in Equation (45) similar to that of Theorem 2 for Equation (17) and its associated vector field in Equation (18).

First, recall the relevant concepts of absolute and relative integral invariant $p$-forms (see, e.g., [12]), a theory started by Poincaré in [56].

Definition 3. We say that a differential $p$-form $\lambda$ in a manifold $M$ is invariant under a vector field $X$ if $\mathcal{L}_{X} \lambda=0$, and an absolute integral invariant of a vector field $X \in \mathfrak{X}(M)$ if $\mathcal{L}_{f} \lambda=0$, for any function $f \in C^{\infty}(M)$, i.e., if $\lambda$ is invariant under the vector field $X$ and $i(X) \lambda=0$, or equivalently, if $i(X) \lambda=0$ and $i(X) d \lambda=0$. Finally, $\lambda$ it is said to be a relative integral invariant if the $(p+1)$-form $d \lambda$ is an absolute integral invariant of $X$, i.e., simply $i(X) d \lambda=0$.

As a well-known example, if $(M, \omega)$ is a symplectic manifold, the 2 -form $\omega$ is an absolute integral invariant of each locally Hamiltonian vector field, and if $\omega$ is exact, $\omega=d \theta$. In the particular case of a 0 -form in $\mathbb{R}^{2}$, i.e., a function $G \in C^{\infty}\left(\mathbb{R}^{2}\right)$, each relative integral invariant function is also an absolute integral invariant function, i.e., the differentiable function $G$ is a first-integral of $X$. Now, the first-integrals of the vector field $\Gamma$, given by Equation (18), are the constants of the motion and the result of Theorem 2 can be reformulated as follows: The function $G(q, v)$ is a relative integral invariant of the vector field $\Gamma$, given by Equation (18), if and only if there exists a Jacobi multiplier $R$ (with respect to the volume form $\Omega=d q \wedge d v$ ) such that $R i(\Gamma) \Omega=d G$. The generalisation to the time-dependent case is as follows: 
Theorem 6. A 1-form $\lambda$ in $\mathbb{R}^{3} \equiv \mathbb{R} \times T \mathbb{R}$ is a relative integral invariant of the SODE vector field (45) if and only if there exists a Jacobi multiplier $R$ for $\Gamma$ (with respect to the volume form $\Omega=d t \wedge d q \wedge d v)$ such that $\operatorname{Ri}(\Gamma) \Omega=d \lambda$.

Proof. If $R$ is a Jacobi multiplier such that $R i(\Gamma) \Omega=d \lambda$, then it trivially follows that $i(\Gamma) d \lambda=0$, i.e., the 1 -form $\lambda$ is a relative integral invariant. Conversely, as by Equation (45), $i(\Gamma) d t=1$, we have that if $\lambda$ is a 1 -form, then $d t \wedge d \lambda$ is a volume form, and from the relation

$$
i(\Gamma)(d t \wedge d \lambda)=(i(\Gamma) d t) d \lambda-d t \wedge(i(\Gamma) d \lambda)=d \lambda-d t \wedge(i(\Gamma) d \lambda),
$$

we see that if $\lambda$ is a relative integral invariant of the vector field $\Gamma$, as $i(\Gamma) d \lambda=0$, we have $i(\Gamma)(d t \wedge d \lambda)=d \lambda$, i.e., the vector field $\Gamma$ is divergence-free with respect to the volume form $d t \wedge d \lambda$, because $\mathcal{L}_{\Gamma}(d t \wedge d \lambda)=d(i(\Gamma)(d t \wedge \lambda))=d(d \lambda)=0$, and the function $R$ such that $d t \wedge d \lambda=R \Omega$ is a Jacobi multiplier for $\Gamma$ with respect to the volume form $\Omega$, because $\mathcal{L}_{\Gamma}(R \Omega)=\mathcal{L}_{R \Gamma} \Omega$.

If the coordinate expression of the 1 -form $\lambda$ on $\mathbb{R}^{3}$ is $\lambda=A d t+B d q+C d v$, for which $d \lambda=\left(B_{t}-A_{q}\right) d t \wedge d q+\left(C_{t}-A_{v}\right) d t \wedge d v+\left(C_{q}-B_{v}\right) d q \wedge d v$, we have $d t \wedge d \lambda=$ $\left(C_{q}-B_{v}\right) \Omega$, and if $\lambda$ is a relative integral invariant of the vector field $\Gamma$, then the function

$$
R=\frac{\partial C}{\partial q}-\frac{\partial B}{\partial v}
$$

is a Jacobi multiplier for $\Gamma$ with respect to the volume form $\Omega$ because $R i(\Gamma) \Omega=d \lambda$.

Note that as

$$
i(\Gamma) d \lambda=\left(B_{t}-A_{q}\right)(d q-v d t)+\left(C_{t}-A_{v}\right)(d v-F d t)+\left(C_{q}-B_{v}\right)(v d v-F d q)
$$

$\lambda$ is a relative integral invariant of $\Gamma$ if and only if

$$
\left\{\begin{array}{l}
v\left(B_{t}-A_{q}\right)+F\left(C_{t}-A_{v}\right)=0 \\
\left(B_{t}-A_{q}\right)-F\left(C_{q}-B_{v}\right)=0 \\
\left(C_{t}-A_{v}\right)+v\left(C_{q}-B_{v}\right)=0
\end{array}\right.
$$

and therefore, assuming that $F v \neq 0$, we can define $R$ equivalently by

$$
R=\frac{1}{F}\left(\frac{\partial B}{\partial t}-\frac{\partial A}{\partial q}\right)=\frac{1}{v}\left(\frac{\partial A}{\partial v}-\frac{\partial C}{\partial t}\right)
$$

As a consequence of the result of this theorem, the search for Jacobi multipliers for $\Gamma$ reduces to that of relative integral invariant 1 -forms.

Corollary 2. Let $G(t, q, v)$ and $K(t, q, v)$ be two functionally independent functions. Then the 1 -form $\lambda=G d K$ is a relative integral invariant of $\Gamma$ if and only if both $G$ and $K$ are constants of the motion, i.e., absolute invariant integrals for $\Gamma$. In this case, the corresponding Jacobi multiplier is

$$
R=\left|\frac{\partial G}{\partial q} \frac{\partial K}{\partial v}-\frac{\partial G}{\partial v} \frac{\partial K}{\partial q}\right|=\left|\frac{\partial(G, K)}{\partial(q, v)}\right|
$$

with associated Lagrangian

$$
L(t, q, v)=\int_{v_{0}}^{v}(v-\zeta)\left|\frac{\partial(G, K)}{\partial(q, v)}\right|_{(t, q, \zeta)} d \zeta+\int_{q_{0}}^{q} F\left(t, \zeta, v_{0}\right)\left|\frac{\partial(G, K)}{\partial(q, v)}\right|_{\left(t, \zeta, v_{0}\right)} d \zeta
$$

Proof. Note first that as the functions $G$ and $K$ are assumed to be functionally independent, then the 1 -form $\lambda=G d K$ is such that $d \lambda=d G \wedge d K$ is different from zero. As $i(\Gamma)(d G \wedge d K)=(i(\Gamma) d G) d K-(i(\Gamma) d K) d G$, the 1 -form $G d K$ is a relative integral invariant if $i(\Gamma) d G=i(\Gamma) d K=0$, i.e., $G$ and $K$ are relative integral invariant, because of the 
functional independence of the functions $G$ and $K$. The expression (51) of the associated Jacobi multiplier $R$ is obtained from Equation (49) by taking into account that, in this case, $A=G K_{t}, B=G K_{q}$ and $C=G K_{v}$. The Lagrangian is obtained by putting Equation (51) into Equation (48).

As the multiplier $R$, determined by an integral invariant, admits other equivalent expressions in Equation (50), in this case, we can write Equation (51) as

$$
\begin{aligned}
& R=\frac{1}{v}\left(\frac{\partial G}{\partial v} \frac{\partial K}{\partial t}-\frac{\partial G}{\partial t} \frac{\partial K}{\partial v}\right)=\frac{1}{v}\left|\frac{\partial(G, K)}{\partial(v, t)}\right|, \\
& R=\frac{1}{F}\left(\frac{\partial G}{\partial t} \frac{\partial K}{\partial q}-\frac{\partial G}{\partial q} \frac{\partial K}{\partial t}\right)=\frac{1}{F}\left|\frac{\partial(G, K)}{\partial(t, q)}\right|,
\end{aligned}
$$

and, consequently, the Lagrangian $L$ can equivalently be written as

$$
\begin{array}{r}
L(t, q, v)=\int_{v_{0}}^{v} \frac{v-\zeta}{\zeta}\left|\frac{\partial(G, K)}{\partial(v, t)}\right|_{(t, q, \zeta)} d \zeta+\int_{q_{0}}^{q} \frac{F\left(t, \zeta, v_{0}\right)}{v_{0}}\left|\frac{\partial(G, K)}{\partial(v, t)}\right|_{\left(t, \zeta, v_{0}\right)} d \zeta, \\
L(t, q, v)=\int_{v_{0}}^{v} \frac{v-\zeta}{F(t, q, \zeta)}\left|\frac{\partial(G, K)}{\partial(t, q)}\right|_{(t, q, \zeta)} d \zeta+\int_{q_{0}}^{q}\left|\frac{\partial(G, K)}{\partial(t, q)}\right|_{\left(t, \zeta, v_{0}\right)} d \zeta,
\end{array}
$$

respectively.

These equivalent forms of the Lagrangian determined by the Jacobi multiplier associated to two independent first-integrals were obtained by Leubner [36]. Other results can be derived from here. Actually, the time-independent Lagrangian Equation (36) generated by a constant of the motion $G(q, v)$ may be obtained from Equation (53) by taking into account that from $G$, we can construct a second constant of the motion $K$ such that $K_{t}=1$, see [36].

\section{More Illustrative Examples}

As a first example, we recall that the classical equation of motion of a damped harmonic oscillator is

$$
\ddot{q}+\lambda \dot{q}+\omega^{2} q=0, \quad \lambda, 0<\omega \in \mathbb{R},
$$

which is described by the vector field in $\mathbb{R} \times T \mathbb{R} \equiv \mathbb{R}^{3}$ given by Equation (45), with $F(q, v)=-\left(\lambda v+\omega^{2} q\right)$,

$$
\Gamma=\frac{\partial}{\partial t}+v \frac{\partial}{\partial q}-\left(\lambda v+\omega^{2} q\right) \frac{\partial}{\partial v}
$$

and then, if we consider the volume form in $\mathbb{R}^{3} \Omega=d t \wedge d q \wedge d v$, div $\Gamma$ is constant, $\operatorname{div} \Gamma=-\lambda$, which shows that [57] $R=e^{\lambda t}$ is a Jacobi multiplier for the vector field $\Gamma$. Consequently, there will exist a Lagrange function $L(t, q, v)$ such that

$$
\frac{\partial^{2} L}{\partial v^{2}}=e^{\lambda t}
$$

Other possible Jacobi multipliers have been found in [57].

More generally, if the vector field $\Gamma$ is such that $\operatorname{div} \Gamma=\mu(t) \in \mathbb{R}$, then the function

$$
R=\exp \left(-\int^{t} \mu\left(t^{\prime}\right) d t^{\prime}\right)
$$

is a Jacobi multiplier because $\Gamma(R)+R \operatorname{div} \Gamma=-\mu R+\mu R=0$. For instance, a slight generalisation of the damped harmonic oscillator is given by the differential equation

$$
\ddot{q}+\psi(t) \dot{q}+\omega^{2} q=0,
$$


which is described by the vector field on $\mathbb{R}^{3}$

$$
\Gamma=\frac{\partial}{\partial t}+v \frac{\partial}{\partial q}-\left(\psi(t) v+\omega^{2} q\right) \frac{\partial}{\partial v},
$$

and if we consider the volume form in $\Omega=d t \wedge d q \wedge d v, \operatorname{div} \Gamma$ is a function of $t, \operatorname{div} \Gamma=-\psi(t)$, and it shows that if $\varphi(t)=\int^{t} \psi(\zeta) d \zeta$, then $e^{\varphi(t)}$ is a Jacobi multiplier for $\Gamma$, which was already found by Jacobi. The corresponding Lagrangian $L$ is obtained from Equation (48) by choosing $v_{0}=0$ and $q_{0}=0$ :

$$
L(t, q, v)=e^{\varphi(t)} \frac{1}{2}\left(v^{2}-\omega^{2} q^{2}\right) .
$$

In the particular case of $\psi(t)=\lambda$, the well-known Lagrangian proposed by Bateman [58] and later on by Caldirola [59] is recovered as a particular case.

To work out more examples, we only need to look for a Jacobi multiplier $R$ for the given mechanical system and apply the general Equation (48) for the Lagrangian. As we do not need to find the general solution of Equation (46), but it suffices to find only particular solutions of Equation (46) for the multiplier. We can impose restrictions on the form of $R$. For instance, when studying a second-order differential equation in normal form $\ddot{q}=F(t, q, \dot{q})$, with an associated vector field (45), the condition for the function $R=\exp \left(-\int^{t} \mu\left(t^{\prime}\right) d t^{\prime}\right)$ to be a Jacobi multiplier reduces to

$$
\frac{\partial F}{\partial v}=\mu(t),
$$

and then $F(t, q, v)=\mu(t) v+\varphi(t, q)$. In this case, the Lagrangian $L(t, q, v)$ for the secondorder differential equation is such that

$$
\frac{\partial^{2} L}{\partial v^{2}}=\exp \left(-\int^{t} \mu\left(t^{\prime}\right) d t^{\prime}\right),
$$

and then $L$ is, up to the addition of a gauge term, of the form

$$
L(t, q, v)=\exp \left(-\int^{t} \mu\left(t^{\prime}\right) d t^{\prime}\right) \frac{v^{2}}{2}+\phi(t, q) .
$$

The function $\phi(t, q)$ is determined by demanding that the Euler-Lagrange equation must be satisfied, yielding $\phi(t, q)=R(t) \int^{q} \varphi(t, \zeta) d \zeta$. Then

$$
L(t, q, v)=R(t)\left(\frac{1}{2} v^{2}+\int^{q} \varphi(t, \zeta) d \zeta\right) .
$$

Of course, this result can be obtained directly from Equation (48).

There are other interesting examples of physical interest. For instance, Emden-Fowlertype equations (see, e.g., $[60,61]$ and references therein)

$$
\ddot{x}+a(t) \dot{x}+b(t) x^{n}=0,
$$

arise in many problems in mathematical physics and astrophysics [62,63]. This is the case of the Lane-Emden equation

$$
\ddot{x}+\frac{k}{t} \dot{x}+c t^{l} x^{n}=0, \quad k, c \in \mathbb{R}, l, n \in \mathbb{N},
$$

or the original equation introduced by Emden, which corresponds to the particular choice $k=2, c=1, l=0$ :

$$
t \ddot{x}+2 \dot{x}+t x^{n}=0,
$$


for which

$$
F(x, v)=-\frac{2}{t} v-x^{n},
$$

and then, in this case, $\mu(t)=-2 / t$, and therefore, there exists a Jacobi multiplier that depends only on $t, R(t)=t^{2}$, and its associated Lagrangian is, up to a gauge term,

$$
L(t, x, v)=t^{2}\left(\frac{1}{2} v^{2}-\frac{x^{n+1}}{n+1}\right) .
$$

Other alternative Jacobi multipliers were determined in [64].

We can also characterise the functions $F$ for which the corresponding SODE vector field admits a Jacobi multiplier $R$ such that it only depends on $t$ and $x$. In such a case, the Jacobi multiplier equation is

$$
\frac{\partial \log R}{\partial t}+v \frac{\partial \log R}{\partial x}+\frac{\partial F}{\partial v}=0,
$$

from which we see that the function $F$ must be of the form $F(t, x, v)=A(t, x) v+B(t, x) v^{2}+$ $C(t, x)$, where

$$
\frac{\partial \log R}{\partial t}=-A, \quad \frac{\partial \log R}{\partial x}=-2 B,
$$

and the integrability condition for the existence of such a Jacobi multiplier $R(t, x)$ is that $\partial A / \partial x=2 \partial B / \partial t$

As a particular instance appearing in General Relativity, let us consider the case of the so-called Buchdahl Equation [65]

$$
\ddot{x}=\frac{3 \dot{x}^{2}}{x}+\frac{\dot{x}}{t},
$$

which corresponds to the particular choice

$$
A=\frac{1}{t}, \quad B=\frac{3}{x}, \quad C(x)=0,
$$

satisfying the compatibility condition for the existence of the Jacobi multiplier, $\partial A / \partial x=$ $0=\partial B / \partial t$. The Jacobi multiplier $R$ is determined by the conditions

$$
\frac{\partial \log R}{\partial t}=-\frac{1}{t}, \quad \frac{\partial \log R}{\partial x}=-\frac{6}{x},
$$

i.e.,

$$
R(t, x)=\frac{1}{t x^{6}},
$$

with the associated Lagrangian given by Equation (48), i.e., up to a gauge term,

$$
L(t, x, v)=\frac{v^{2}}{2 t x^{6}} .
$$

As a second example of this type, let us consider the SODE [66]:

$$
t x \ddot{x}+x \dot{x}+t(2 x+1) \dot{x}^{2}=0 .
$$

which corresponds to the choice

$$
A=-\frac{1}{t}, \quad B=-\frac{2 x+1}{x}, \quad C(x)=0 .
$$


and therefore, the compatibility condition for the existence of the Jacobi multiplier is satisfied because $\partial A / \partial x=0=\partial B / \partial t$. The Jacobi multiplier $R$ is determined by the conditions

$$
\frac{\partial \log R}{\partial t}=\frac{1}{t}, \quad \frac{\partial \log R}{\partial x}=2 \frac{2 x+1}{x},
$$

with the solution

$$
R(t, x)=t x^{2} e^{4 x},
$$

and associated Lagrangian given by Equation (48), i.e., up to a gauge term,

$$
L(t, x, v)=\frac{1}{2} t x^{2} e^{4 x} v^{2} .
$$

We can use this example to illustrate the more involved procedure based on the corollary of Theorem 6. First of all, observe that, after multiplying Equation (62) by $e^{2 x}$, it is reduced to the equivalent form $d\left(e^{2 x}+x \dot{x}\right) / d t=0$ so that

$$
G_{1}(t, x, v)=e^{2 x} t x v
$$

is a constant of the motion. Another constant of the motion is obtained by solving the equation $e^{2 x} t x \dot{x}=$ const for $\dot{x}$ and integrating with respect to time,

$$
K(t, x, v)=\log t-\frac{2 x-1}{4 t x v} .
$$

Using, for a matter of convenience, the constant of the motion $G=\log G_{1}$ instead of $G_{1}$, we obtain, according to Equation (53), the Jacobi multiplier

$$
R=\frac{1}{v}\left|\frac{\partial(G, K)}{\partial(v, t)}\right|=\frac{1}{t v^{2}},
$$

with the associated Lagrangian Equation (48)

$$
L(t, x, v)=-\frac{1}{t} \log \left(e^{2 x} x v\right),
$$

as obtained by choosing suitable values for the constants of integration and eliminating the gauge terms in Equation (48). Remark that the quotient of both Jacobi multipliers is $G_{1}^{2}$, i.e., a constant of the motion, as expected.

\section{Infinitesimal Symmetries and Jacobi Multipliers}

Our emphasis in the theory of Jacobi multipliers was in its applications to the inverse problem of mechanics, mainly for the interest in the Lagrangian formulation as a first step in the process of obtaining an appropriate Hamiltonian formulation for its quantisation, but we do not forget that the concept of the Jacobi (last) multiplier was introduced to integrate a given system by quadratures. More specifically, the result obtained by Jacobi can be summarised as follows (see [55] for a recent geometric presentation):

If a Jacobi multiplier $\mu$ and a set of $(n-2)$ functionally independent first-integrals, $\left\{I_{1}, \ldots, I_{n-2}\right\}$, for a given vector field $X$ on an oriented $n$-dimensional manifold $(M, \Omega)$ are known, the determination of the integral curves of the vector field $X$ is reduced to quadratures.

In this and the next section, we point out other interesting results concerning the integrability by quadratures that have not been considered from a geometric perspective.

As it has been shown, the usefulness of the constants of motion in the theory of Jacobi multipliers, and quite often, these first-integrals are related to infinitesimal symmetries, one can guess that the infinitesimal symmetries can also play a rôle in the theory. We first start with the simpler 2-dimensional case to recall a celebrated theorem by Lie.

In the simple 2-dimensional case, there is a clear relationship between the existence of an infinitesimal symmetry $Y$ and the existence of a Jacobi multiplier because, given such a 
vector field $Y$, the function $R=(i(Y) i(X) \omega)^{-1}$ is a Jacobi multiplier for $X$, with respect to the volume form $\omega$. In fact, the main property relating Lie symmetries and integrating factors is due to Lie [67]:

Theorem 7. (Lie) Let be $Y \in \mathfrak{X}\left(\mathbb{R}^{2}\right)$ and $\alpha \in \Lambda^{1}\left(\mathbb{R}^{2}\right)$ such that $i(Y) \alpha$ is a nonvanishing function. Then $R=[i(Y) \alpha]^{-1}$ is an integrating factor for $\alpha$ if and only if the vector field $Y$ is an infinitesimal symmetry of the distribution defined by the 1-form $\alpha$, i.e., $\mathcal{L}_{Y} \alpha=f \alpha$ with $f \in C^{\infty}\left(\mathbb{R}^{2}\right)$.

Proof. We can take into account that

$$
d\left[\frac{\alpha}{i(Y) \alpha}\right]=-\frac{d[i(Y) \alpha]}{[i(Y) \alpha]^{2}} \wedge \alpha+\frac{d \alpha}{i(Y) \alpha},
$$

which can also be written as

$$
d\left[\frac{\alpha}{i(Y) \alpha}\right]=\frac{1}{[i(Y) \alpha]^{2}}\left[\left(-\mathcal{L}_{Y} \alpha+i(Y) d \alpha\right) \wedge \alpha+(i(Y) \alpha) d \alpha\right],
$$

where use has been made of $d(i(Y) \alpha)=\mathcal{L}_{Y} \alpha-i(Y) d \alpha$.

Now, as $\alpha$ is a 1 -form,

$$
i(Y)(\alpha \wedge d \alpha)=(i(Y) \alpha) d \alpha+(i(Y) d \alpha) \wedge \alpha,
$$

and as $\alpha \wedge d \alpha$ is a 3 -form, it must be equal to zero, and then

$$
d\left[\frac{\alpha}{i(Y) \alpha}\right]=-\frac{1}{[i(Y) \alpha]^{2}}\left(\left(\mathcal{L}_{Y} \alpha\right) \wedge \alpha\right) .
$$

Therefore, $\alpha /(i(Y) \alpha)$ is closed if and only if $\left(\mathcal{L}_{Y} \alpha\right) \wedge \alpha=0$, i.e., $\mathcal{L}_{Y} \alpha$ is proportional to $\alpha$.

Another proof is as follows: If $X$ is a vector field such that $\alpha(X)=0$, then $X$ is linearly independent of $Y$ at each point because $\alpha(Y)$ is a nonvanishing function. Then, the 1 -form $\beta=\alpha /(i(Y) \alpha)$ is such that $\beta(X)=0$ and $\beta(Y)=1$ by construction. Therefore,

$$
d \beta(X, Y)=X \beta(Y)-Y \beta(X)+\beta([X, Y])=\beta([X, Y]) .
$$

Here, we see that $d \beta=0$ if and only if $\beta([X, Y])=0$, which is equivalent to $\alpha([X, Y])=$ 0 . However, as $\left(i(X) \circ \mathcal{L}_{Y}-\mathcal{L}_{Y} \circ i(X)\right) \alpha=i([X, Y]) \alpha=0$, and $\alpha(X)=0$, we obtain that $i(X) \mathcal{L}_{Y} \alpha=0$, i.e., the vector field $Y$ is a symmetry of the distribution defined by $\alpha$.

We remark that if the vector field $Y$ is a strict symmetry of $X$, i.e., $[X, Y]=0$, then $Y$ is an infinitesimal symmetry of the distribution defined by the 1-form $\alpha$, and the result of the theorem follows, but the result is also valid in the more general case in which there exists a function $\lambda$ such that $[X, Y]=\lambda X$. These vector fields $Y \in \mathfrak{X}(M)$ that are symmetries of the distribution generated by $X$ are sometimes called normalizers of $X$.

In this particular case, we are considering $\alpha$ is the 1 -form $\alpha=i(X) \omega$. The associated first-integral, as indicated in Theorem 1, is the (locally defined) function $F$ such that

$$
\frac{\alpha}{i(Y) \alpha}=d F,
$$

and the function $F$ can be found by one quadrature.

The generalisation of this theorem to higher dimensional manifolds can be found in $[68,69]$ :

Theorem 8. If $n$ vector fields $X_{1}, \ldots, X_{n}$, which are linearly independent at each point of an open set $U \subset \mathbb{R}^{n}$, generate a solvable Lie algebra and are such that $\left[X_{1}, X_{i}\right]=\lambda_{i} X_{1}$ with $\lambda_{i} \in \mathbb{R}$, then the differential equation $\dot{x}_{i}=X_{1} x_{i}$ is solvable by quadratures in $U$. 
However, the main theorem that extends to the $n$-dimensional case, the Lie results for the construction of the Jacobi multiplier when $(n-1)$ infinitesimal symmetries are known in $[67,70]$ :

Theorem 9. Let $\left\{Y_{1}, \ldots, Y_{n-1}\right\}$ be a set of $n-1$ vector fields whose values are linearly independent at each point of the $n$-dimensional oriented manifold $(M, \Omega)$ and are normalizers of the vector field $X \in \mathfrak{X}(M)$. If the function $h=i\left(Y_{1}\right) \cdots i\left(Y_{n-1}\right) i(X) \Omega$ is a nonvanishing function, then $h^{-1}$ is a Jacobi multiplier for the vector field $X \in \mathfrak{X}(M)$.

Proof. We first recall that for any vector field $Y \in \mathfrak{X}(M)$ and any form $\beta \in \Lambda(M)$, we have that

$$
\mathcal{L}_{X} i(Y) \beta=i(Y) \mathcal{L}_{X} \beta+i([X, Y]) \beta,
$$

and if we apply this relation with $Y=Y_{1}$ and $\beta=i\left(Y_{2}\right) \cdots i\left(Y_{n-1}\right) i(X) \Omega$, we obtain that

$$
\mathcal{L}_{X} h=i\left(Y_{1}\right) \mathcal{L}_{X}\left[i\left(Y_{2}\right) \cdots i\left(Y_{n-1}\right) i(X) \Omega\right]+i\left(\left[X, Y_{1}\right]\right) i\left(Y_{2}\right) \cdots i\left(Y_{n-1}\right) i(X) \Omega .
$$

The second term of the right hand side vanishes because $\left[Y_{1}, X\right]$ is proportional to $X$ and $i(X) i(X) \Omega=0$, and therefore, only the first term remains:

$$
X h=\mathcal{L}_{X} h=i\left(Y_{1}\right) \mathcal{L}_{X}\left[i\left(Y_{2}\right) \cdots i\left(Y_{n-1}\right) i(X) \Omega\right] .
$$

Iterating the process, with the vector fields $Y_{2}$, then $Y_{3}$, and so on, we will arrive to

$$
\mathcal{L}_{X} h=i\left(Y_{1}\right) \cdots i\left(Y_{n-1}\right) \mathcal{L}_{X} i(X) \Omega
$$

Now, as $\mathcal{L}_{X} i(X) \Omega=i(X) \mathcal{L}_{X} \Omega=\operatorname{div} X i(X) \Omega$, we find that

$$
X h=\operatorname{div} X i\left(Y_{1}\right) \cdots i\left(Y_{n-1}\right) i(X) \Omega=h \operatorname{div} X
$$

Finally, remark that if $h$ is a nonvanishing function such that $X h=h \operatorname{div} X$, then the function $R=h^{-1}$ satisfies the Jacobi multiplier condition.

Note that if we use coordinates such that $\Omega=d x^{1} \wedge \cdots d x^{n}$, then $h$ is, up to a real factor, equal to

$$
\bar{h}=\left|\begin{array}{cccc}
X^{1} & \cdots & \cdots & X^{n} \\
Y_{1}^{1} & \cdots & \cdots & Y_{1}^{n} \\
\vdots & \vdots & \vdots & \vdots \\
Y_{n-1}^{1} & \cdots & \cdots & Y_{n-1}^{n}
\end{array}\right|
$$

where $X^{i}$ denote the components of the vector field $X$ and $Y_{\alpha}^{i}$ the components of the vector field $Y_{\alpha}$.

This result has been presented in [64,71-74].

\section{Jacobi Multipliers and Hojman Symmetry}

Apart from the usual way of finding first-integrals from infinitesimal symmetries via the Noether theorem, and a second procedure based on the existence of alternative geometric structures for the description of the vector field providing us a recursion operator, there is a third approach started by Hojman [75] and González-Gascón in [76], which is becoming more and more important because of its applications in $f(R)$-gravity and FRW cosmology [77-83]. It was introduced first for divergence-free vector fields in an oriented manifold $(M, \Omega)$, in the particular case of a SODE and then generalised to arbitrary SODE vector fields. The general result is a consequence of the fundamental relation in Equation (8). The geometric approach to this generalised Hojman symmetry was recently given in [84]. As a consequence of relation in Equation (8), if the vector field $X \in \mathfrak{X}(M)$ in an oriented manifold $(M, \Omega)$ is divergence-free and the vector field $Y$ is an infinitesimal symmetry of $X$, i.e., $[X, Y]=0$, we have that $\operatorname{div}(Y)$ is a constant of the motion for $X$. When, instead, the 
vector field $Y$ is an infinitesimal symmetry of the 1-dimensional distribution generated by $X$, i.e., there exists a function $h$ such that $[Y, X]=h X$, we have that the function $\operatorname{div}(Y)+h$ is a constant of the motion for $X$. Actually, if $\operatorname{div}(X)=0$, then $\mathcal{L}_{X} \Omega=0$, and hence, as $[X, Y]=-h X$,

$$
\mathcal{L}_{X}\left(\mathcal{L}_{Y} \Omega\right)=\mathcal{L}_{Y}\left(\mathcal{L}_{X} \Omega\right)+\mathcal{L}_{-h X} \Omega=-\mathcal{L}_{X}(h \Omega)=-X(h) \Omega,
$$

and therefore, as $\mathcal{L}_{X}\left(\mathcal{L}_{Y} \Omega\right)=\mathcal{L}_{X}(\operatorname{div}(Y) \Omega)=\mathcal{L}_{X}(\operatorname{div}(Y)) \Omega$, we obtain that $\mathcal{L}_{X}(\operatorname{div}(Y)+h)=0$, and therefore, the following function is a constant of the motion $I$ for $X$ :

$$
I=\operatorname{div}(Y)+h
$$

If the vector field is not divergence-free, we can use the Jacobi multiplier theory, and as for any nonvanishing function $R$, the constants of motion of $X$ coincide with those of $R X$, in the particular case of a Jacobi multiplier $R$ for $X$, the vector field $\bar{X}=R X$ is such that when $[Y, X]=h X$, we have that $[Y, \bar{X}]=\bar{h} \bar{X}$ with $\bar{h}=(Y(R) / R)+h$, and hence, we have the constant of motion for $\bar{X}$ and, therefore, for $X$, given by

$$
I=\operatorname{div}(Y)+\bar{h}=\operatorname{div}(Y)+Y(\log R)+h .
$$

These general results can be applied to specific examples, and we recover as particular examples many previously found constants of motion (see [84]). For instance, one can apply the general theory to both Hamiltonian and Lagrangian formulations of autonomous systems or generic second-order differential equations. In this respect, the Jacobi multiplier given by the determinant of the Hessian matrix $W$ in the Lagrangian case explains the result obtained by Lutzky [85] as a particular case of the constant of motion given by Equation (66).

In the case of nonautonomous systems, as the dynamical vector fields must be replaced by 1-dimensional distributions, the more general condition $[Y, X]=h X$, which means that the vector field $Y$ preserves the 1-dimensional distribution generated by $X$ is relevant in the context of symmetry for such systems. We can study particular examples of non-autonomous systems of first-order differential equations $[75,76]$ and also Hamiltonian systems as in [86], non-autonomous systems of second-order differential equations $[75,76,87,88]$ and, in particular, systems admitting a Lagrangian formulation $[85,88]$.

\section{Conclusions}

A geometrical approach to the theory of Jacobi multipliers has been developed: after introducing the usual definition, it was pointed out that the search for Jacobi multipliers is equivalent to the determination of invariant volume-forms.

As a first application of the general theory, we used this concept in the inverse problem of classical mechanics because, as it has been shown in Section 3, when a Jacobi multiplier for a given second-order differential equation is known, it it possible to find a Lagrangian formulation for such a system. This is particularly interesting in the quantization process. Of course, such Lagrangians, which can be found by means of two quadratures, are, in general, non-standard Lagrangians, as has been illustrated with several examples. The Lagrangian case for autonomous $n$-dimensional systems has also been considered, and it was shown that the determinant of the Hessian matrix is a Jacobi multiplier. The theory has been extended to the non-autonomous case where a similar result holds, and an explicit formula for the determination of a Lagrangian for the particular case of a non-autonomous second-order differential equation is given. The bijection between firstintegrals of 2-dimensional systems and Jacobi multipliers is then extended to the case of a non-autonomous second-order differential equation as a bijection between relative integral invariants on one side and Jacobi multipliers on the other. Several interesting examples illustrate the theory. 
As a second application, the relation for 2-dimensional systems among infinitesimal symmetries of the distribution generated by the given vector field $X$ and its Jacobi multipliers is extended in one direction: it is shown how it is possible to obtain a Jacobi multiplier when $(n-1)$ infinitesimal symmetries of the distribution generated by $X$ are known. Finally, the relevance of Jacobi multipliers in the search for first integrals of a vector field $X$, i.e., the recently developed geometric approach to Hojman symmetry, is summarised. In fact, a Jacobi multiplier for the vector field $X$ allows us to use a conformally related divergence-free vector field instead of $X$, and then, the infinitesimal symmetries of this one provide us first integrals for $X$.

Author Contributions: Investigation, J.F.C. and J.F.-N. Both authors have read and agreed to the published version of the manuscript.

Funding: This research was supported by the Spanish Ministerio de Ciencia, Innovación y Universidades project PGC2018-098265-B-C31.

Conflicts of Interest: The authors declare no conflict of interest.

\section{References}

1. Whittaker, E.T. A Treatise on the Analytical Dynamics of Particles and Rigid Bodies: With an Introduction to the Problem of Three Bodies; Cambridge University Press: Cambridge, UK, 1989.

2. Helmholtz, H. Über die physikalische Bedeutung des Prinzips der kleinsten Wirking. J. Reine Angew. Math. 1887, 100, 137-141.

3. Douglas, J. Solution of the inverse problem of the calculus of variations. Trans. Am. Math. Soc. 1941, 50, 71-128. [CrossRef]

4. Crampin, M. On the differential geometry of the Euler-Lagrange equations and the inverse problem in Lagrangian dynamics. $J$. Phys. A Math. Gen. 1981, 14, 2567-2575. [CrossRef]

5. Sarlet, W. The Helmholtz conditions revisited. A new approach to the inverse problem of Lagrangian dynamics. J. Phys. A Math. Gen. 1982, 15, 1503-1517. [CrossRef]

6. Currie, D.G.; Saletan, E.J. q-equivalent particle Hamiltonians. The classical one-dimensional case. J. Math. Phys. 1966, 7, 967-974. [CrossRef]

7. Hojman, S.; Harleston, H. Equivalent Lagrangians: multidimensional case. J. Math. Phys. 1981, 22, 1414-1419. [CrossRef]

8. nena, J.F.C.; Ibort, L.A. Non-Noether constants of motion. J. Phys. A Math. Gen. 1983, 16, 1-7.

9. Abraham, R.; Marsden, J.E. Foundations of Mechanics, 2nd. ed.; Benjamin: Reading, MA, USA, 1978.

10. Crampin, M. Tangent bundle geometry for Lagrangian dynamics. J. Phys. A Math. Gen. 1983, 16, 3755-3772. [CrossRef]

11. M, M.C.; Pirani, F.A.E. Applicable Differential Geometry; University Press: Cambridge, UK, 1986.

12. Libermann, P.; Marle, C.-M. Symplectic Geometry and Analytical Mechanics; D. Reidel Pub. Co.: Dordrecht, The Netherlands, 1987.

13. Cariñena, J.F.; Rañada, M.F.; Santander, M. Lagrangian formalism for nonlinear second-order Riccati systems: one-dimensional integrability and two-dimensional superintegrability. J. Math. Phys. 2005, 46, 062703. [CrossRef]

14. Cariñena, J.F.C.; P, P.G.; Rañada, M.F. Higher-order Abel equations: Lagrangian formalism, first integrals and Darboux polynomials. Nonlinearity 2009, 22, 2953-2969. [CrossRef]

15. Musielak, Z.E. Standard and non-standard Lagrangians for dissipative dynamical systems with variable coefficients. J. Phys. A Math. Theor. 2008, 41, 055205. [CrossRef]

16. Musielak, Z.E.; Roy, D.; Swift, L.D. Method to derive Lagrangian and Hamiltonian for a nonlinear dynamical system with variable coefficients. Chaos Solitons Fractals 2008, 38, 894-902. [CrossRef]

17. Musielak, Z.E. General conditions for the existence of non-standard Lagrangians for dissipative dynamical systems. Chaos Solitons Fractals 2009, 42, 2645-2652. [CrossRef]

18. Cieśliński, J.L.; Nikiciuk, T. A direct approach to the construction of standard and non-standard Lagrangians for dissipative-like dynamical systems with variable coefficients. J. Phys. A Math. Theor. 2010, 43, 175205. [CrossRef]

19. El-Nabulsi, R.A. Nonlinear dynamics with non-standard Lagrangians. Qual. Theory Dyn. Syst. 2013, 12, 273-291. [CrossRef]

20. Saha, A.; Talukdar, B. On the non-standard Lagrangian equations. arXiv 2013, arXiv:1301.2667.

21. Cariñena, J.F.; Guha, P.; Rañada, M.F. A geometric approach to higher-order Riccati chain: Darboux polynomials and constants of the motion. J. Phys. Conf. Ser. 2009, 175, 012009. [CrossRef]

22. Jacobi, C.G.J. Sur le principe du dernier multiplicateur et sur son usage comme nouveau principe général de mécanique. J. Math. Pures Appl. 1845, 10, 337-346.

23. Jacobi, C.G.J. Jacobi's Lectures on Dynamics, 2nd ed.; Clebsch, A., Ed.; Hindustan Book Agency: New Delhi, Indea, 2009.

24. Nucci, M.C.; Leach, P.G.L. The Jacobi's Last Multiplier and its applications in mechanics. Phys. Scr. 2008, 78, 065011. [CrossRef]

25. Nucci, M.C.; Leach, P.G.L. An old method of Jacobi to find Lagrangians. J. Nonlin. Math. Phys. 2009, 16, 431-441. [CrossRef]

26. Nucci, M.C.; Tamizhmani, K.M. Lagrangians for dissipative nonlinear oscillators: The method of Jacobi last multiplier. J. Nonlinear Math. Phys. 2010, 17, 167-178. [CrossRef] 
27. Chen, Y.; Liu, Q.; Su, H. Generalized Hamiltonian forms of dissipative mechanical systems via a unified approach. J. Geom. Phys. 2021, 160, 103976. [CrossRef]

28. Negri, L.J.; Oliveira, L.C.; Teixeira, J.M. Canonoid transformations and constants of motion. J. Math. Phys. 1987, 28, 2369-2372. [CrossRef]

29. Jacobi, C.G.J. Sul principio dell'ultimo moltiplicatore, e suo uso come nuovo principio generale di meccanica. Giornale Arcadico di Scienze Lettere ed Arti 1844, 99, 129-146.

30. Jacobi, C.G.J. Theoria novi multiplicatoris systemati aequationum differentialium vulgarium applicandi. J. Reine Angew. Math. 1844, 27, 199-268; Reprint in 1845, 29, 213-279+333-376.

31. Crasmareanu, M. Last multipliers on manifolds. Tensor 2005, 66, 18-25.

32. Crasmareanu, M. Last multipliers for Riemannian geometries, Dirichlet forms and Markov diffusion semigroups. J. Geom. Anal. 2017, 27, 2618-2643. [CrossRef]

33. Crasmareanu, M. Last multipliers for multivectors with applications to Poisson geometry. Taiwan. J. Math. 2009, 3, 1623-1636. [CrossRef]

34. Kobussen, J.A. Some Comments on the Lagrangian Formalism for Systems with General Velocity Dependent Forces. Acta Phys. Austr. 1979, 51, 293-309.

35. Okubo, S. Canonical quantization of some dissipative systems and nonuniqueness of Lagrangians. Phys. Rev. A 1981, 23, 2776-2784. [CrossRef]

36. Leubner, C. Inequivalent Lagrangians from constants of motion. Phys. Lett. A 1981, 86, 68-70. [CrossRef]

37. López, G. One-Dimensional Autonomous Systems and Dissipative Systems. Ann. Phys. 1996, 251, 372-383. [CrossRef]

38. López, G. Hamiltonians and Lagrangians for N-dimensional autonomous systems. Ann. Phys. 1996, 251, 363-371. [CrossRef]

39. González-Gascón, F. Divergence-free vector fields and integration via quadratures. Phys. Lett. A 1996, 225, 269-273. [CrossRef]

40. Yan, C.C. Construction of Lagrangians and Hamiltonians from the equation of motion. Am. J. Phys. 1978, 46, 671-675. [CrossRef]

41. Casetta, L.; Pesce, C.P. The inverse problem of Lagrangian mechanics for Meshchersky's equation. Acta Mech. 2014, 225, 1607-1623. [CrossRef]

42. Cariñena, J.F.; Guha, P. Non-standard Hamiltonian structures of Liénard equation and contact geometry. Int. J. Geom. Methods Mod. Phys. 2019, 16, 1940001. [CrossRef]

43. Liénard, A. Étude des oscillations entretenues. Revue Générale de l'Électricité 1928, 23, 901-912; Ibid. 946-954.

44. Chiellini, A. Sull'integrazione dell'equazione differenziale $y^{\prime}+P y^{2}+Q y^{3}=0$. Boll. Unione Mat. Ital. 1931, 10, $301-307$.

45. Ince, E.L. Ordinary Differential Equations; Dover: New York, NY, USA, 1956.

46. Gubbiotti, G.; Nucci, M.C. Noether symmetries and the quantization of a Liénard-type nonlinear oscillator. J. Nonlinear Math. Phys. 2014, 21, 248-264. [CrossRef]

47. Ruby, V.C.; Senthilvelan, M.; Lakshmanan, M. Exact quantization of a PT-symmetric (reversible) Liénard-type nonlinear oscillator. J. Phys. A Math. Theor. 2014, 45, 382002. [CrossRef]

48. Chandrasekar, V.K.; Senthilvelan, M.; Lakshmanan, M. On the general solution for the modified Emden-type equation $\ddot{x}+\alpha x \dot{x}+$ $\beta x^{3}=0$. J. Phys. A Math. Theor. 2007, 40,4717-4727. [CrossRef]

49. Mathews, P.M.; Lakshmanan, M. On a unique nonlinear oscillator. Quart. Appl. Math. 1974, 32, 215-218. [CrossRef]

50. Cariñena, J.F.; Rañada, M.F.; Santander, M.; Senthilvelan, M. A non-linear Oscillator with quasi-Harmonic behaviour: two- and n-dimensional Oscillators. Nonlinearity 2004, 17, 1941-1963. [CrossRef]

51. Cariñena, J.F.; Rañada, M.F. ; Santander, M. One-dimensional model of a quantum non-linear Harmonic Oscillator. Rep. Math. Phys. 2004, 54, 285-293. [CrossRef]

52. Bagchi, B.; Ghosh, S.; Pal, B.; Poriad, S. Qualitative analysis of certain generalized classes of quadratic oscillator systems. J. Math. Phys. 2016, 57, 022701. [CrossRef]

53. Cariñena, J.F.; Ibort, A.; Marmo, G.; Morandi, G. Geometry from Dynamics: Classical and Quantum; Springer: Dordrecht, The Netherlands, 2015.

54. Crampin, M.; Thompson, G. Affine bundles and integrable almost tangent structures. Math. Proc. Camb. Phil. Soc. 1985, 98, 61-71. [CrossRef]

55. Cariñena, J.F.; Santos, P. Jacobi Multipliers and Hamel's formalism. J. Phys. A Math. Theor. 2021, 54, 225203. [CrossRef]

56. Poincaré, H. Les Méthodes Nouvelles de la Méchanique C'eleste; Gauthier-Villars: Paris, France, 1892; Volume 3.

57. Nucci, M.C.; Leach, P.G.L. Lagrangians galore. J. Math. Phys. 2007, 48, 123510. [CrossRef]

58. Bateman, H. On dissipative systems and related variational principles. Phys. Rev. 1931, 38, 815-819. [CrossRef]

59. Caldirola, P. Forze non conservative nella meccanica quantistica. Nuovo Cim. 1941, 18, 393-400. [CrossRef]

60. Cariñena, J.F.; Leach, P.G.L.; de Lucas, J. Quasi-Lie schemes and Emden-Fowler equations. J. Math. Phys. 2009, 50, 103515. [CrossRef]

61. Guha, P. Generalized Emden-Fowler equations in noncentral curl forces and first integrals. Acta Mech. 2020, 231, 815-825. [CrossRef]

62. Chandrasekhar, S. Principles of Stellar Dynamics; Univ. Chicago Press: Chicago, IL, USA, 1942.

63. Chandrasekhar, S. An Introduction to the Study of Stellar Structure; Dover: New York, NY, USA, 1957.

64. Nucci, M.C.; Leach, P.G.L. Jacobi's last multiplier and symmetries for the Kepler problem plus a lineal story. J. Phys. A Math. Gen. 2004, 37, 7743-7753. [CrossRef]

65. Buchdahl, H.A. A relativistic fluid sphere resembling the Emden polytrope of index 5. Astrophys. J. 1964, 140, 1512-1515. [CrossRef] 
66. Duarte, L.G.S.; Duarte, S.E.S.; da Mota, A.C.P.; Skea, J.E.F. Solving the second-order ordinary differential equations by extending the Prelle-Singer method. J. Phys. A Math. Gen. 2001, 34, 3015-3024. [CrossRef]

67. Lie, S. Vorlesungen über Differentialgleichungen mit Bekannten Infinitesimalen Transformationen; B.G. Teubner: Leipzig, Germany, 1912.

68. Cariñena, J.F.; Falceto, F.; Grabowski, J.; Rañada, M.F. Geometry of Lie integrability by quadratures. J. Phys. A Math. Theor. 2015, 48, 215206. [CrossRef]

69. Cariñena, J.F.; Falceto, F.; Grabowski, J. Solvability of a Lie algebra of vector fields implies their integrability by quadratures. J. Phys. A Math. Theor. 2016, 49, 425202. [CrossRef]

70. Lie, S. Veralgemeinerung und neue Verwerthung der Jacobischen Multiplicator-Theorie. Christ. Forh. 1874, 255-274.

71. Nucci, M.C. Jacobi Last Multiplier and Lie Symmetries: A Novel Application of an Old Relationship. J. Nonl. Math. Phys. 2005, 12, 284-304. [CrossRef]

72. Nucci, M.C. What symmetries can do for you. Int. J. Modern Phys. Conf. Ser. 2015, 38, 1560076. [CrossRef]

73. Nucci, M.C. Symmetries, Lagrangians and Conservation Laws of an Easter Island Population Model. Symmetry 2015, 7, 1613-1632. [CrossRef]

74. Nucci, M.C. The nonlinear pendulum always oscillates. J. Nonlin. Math. Phys. 2017, 24, 146-156. [CrossRef]

75. Hojman, S. A new conservation law constructed without using either Lagrangians or Hamiltonians. J. Phys. A Math. Gen. 1992, 25, L291-L295. [CrossRef]

76. González-Gascón, F. Geometric foundations of a new conservation law discovered by Hojman. J. Phys. A Math. Gen. 1994, 27, L59-L60. [CrossRef]

77. Darabi, F.; Golmohammadi, M.; Rezaei-Aghdam, A. FRW string cosmological solutions via Hojman symmetry. Int. J. Geom. Methods Mod. Phys. 2020, 17, 2050175. [CrossRef]

78. Darabi, F.; Golmohammadi, M.; Rezaei-Aghdam, A. Generalized ( $2+1)$-dimensional BTZ black holes via Hojman symmetry. arXiv 2021, arXiv:2010.08424v2.

79. Wei, H.; Zhou, Y.N.; Li, H.Y.; Zou, X.B. Hojman symmetry in $f(T)$ theory, Astrophys. Space Sci. 2015, 360, 1-7

80. Wei, H.; Zhou, Y.N.; Li, H.Y.; Zou, X.B. Exact cosmological solutions of $f(R)$ theories via Hojman symmetry. Nucl. Phys. B 2016, 903, 132-149. [CrossRef]

81. Capozziello, S.; Roshanc, M. Exact cosmological solutions from Hojman conservation quantities. Phy. Lett. B 2013, 726, 471-480. [CrossRef]

82. Paolella, M.C.; Capozziello, S. Hojman symmetry approach for scalar-tensor cosmology. Phy. Lett. A 2015, 379, 1304-1308. [CrossRef]

83. Paliathanasis, A.; Leach, P.G.L.; Capozziello, S. On the Hojman conservation quantities in Cosmology. Phy. Lett. B 2016, 755, 8-12. [CrossRef]

84. Cariñena, J.F.; Rañada, M.F. Jacobi multipliers and Hojman symmetry. Int. J. Geom. Methods Mod. Phys. 2021, $18,2150166$.

85. Lutzky, M. Remarks on a recent theorem about conserved quantities. J. Phys. A: Math. Gen. 1995, 28, L637-L638. [CrossRef]

86. Gu, S.L.; Wei, K.X. Study on The Symmetry and Conserved Quantities for Hamilton Systems. In Proceedings of the International Conference on Logistics Engineering, Management and Computer Science (LEMCS 2014), Shenyang, China, 24-26 May 2014; Advances in Intelligent Systems Research Series; pp. 798-801.

87. González-Gascón, F. Notes on the Connection between the Symmetries and the First Integrals of Dynamical Systems. Lett. Nuovo Cim. 1977, 19, 366-368. [CrossRef]

88. Zhang, H.B.; Chen, L.Q. The Unified Form of Hojman's Conservation Law and Lutzky's Conservation Law. J. Phys. Soc. Jpn. 2005, 74, 905-909. [CrossRef] 